\title{
The axion-baryon coupling in SU(3) heavy baryon chiral perturbation theory
}

\author{
Thomas Vonk, ${ }^{a}$ Feng-Kun Guo ${ }^{b, c}$ and Ulf-G. Meißner ${ }^{a, d, e}$ \\ ${ }^{a}$ Helmholtz-Institut für Strahlen- und Kernphysik and Bethe Center for Theoretical Physics, \\ Universität Bonn, Bonn D-53115, Germany \\ ${ }^{b}$ CAS Key Laboratory of Theoretical Physics, Institute of Theoretical Physics, \\ Chinese Academy of Sciences, Beijing 100190, China \\ ${ }^{c}$ School of Physical Sciences, University of Chinese Academy of Sciences, \\ Beijing 100049, China \\ ${ }^{d}$ Institute for Advanced Simulation, Institut für Kernphysik and Jülich Center for Hadron Physics, \\ Forschungszentrum Jülich, Jülich D-52425, Germany \\ e Tbilisi State University, Tbilisi 0186, Georgia \\ E-mail: vonk@hiskp.uni-bonn.de, fkguo@itp.ac.cn, \\ meissner@hiskp.uni-bonn.de
}

ABSTRACT: In the past, the axion-nucleon coupling has been calculated in the framework of $\mathrm{SU}(2)$ heavy baryon chiral perturbation theory up to third order in the chiral power counting. Here, we extend these earlier studies to the case of heavy baryon chiral perturbation theory with $\mathrm{SU}(3)$ flavor symmetry and derive the axion coupling to the full $\mathrm{SU}(3)$ baryon octet, showing that the axion also significantly couples to hyperons. As studies on dense nuclear matter suggest the possible existence of hyperons in stellar objects such as neutron stars, our results should have phenomenological implications related to the so-called axion window.

KeYwords: Chiral Lagrangians, Cosmology of Theories beyond the SM, Effective Field Theories

ArXiv EPrint: 2104.10413 


\section{Contents}

1 Introduction 1

$2 \mathrm{SU}(3)$ chiral perturbation theory with axions: general remarks $\quad 4$

2.1 Ingredients from the axion-quark interaction Lagrangian 4

$\begin{array}{ll}2.2 & \text { Building blocks of the chiral Lagrangian }\end{array}$

2.3 General form of the axion-baryon coupling 9

3 Determining the axion-baryon coupling $\quad 10$

$\begin{array}{ll}3.1 \text { Leading order: tree level contributions } & 10\end{array}$

$\begin{array}{lll}3.2 & \text { Expansion in the baryon mass } & 11\end{array}$

$\begin{array}{lll}3.3 & \text { Next-to-next-to-leading order: contributions from } \chi_{-} & 12\end{array}$

$\begin{array}{lll}3.4 & \text { Next-to-next-to-leading order: contributions from } \chi_{+} & 13\end{array}$

$\begin{array}{ll}3.5 & \text { Next-to-next-to-leading order: counter-terms } \\ 3.6 & 14\end{array}$

$\begin{array}{lll}3.6 & \text { Loops } & 15\end{array}$

$\begin{array}{lll}3.6 .1 & \text { Diagram (a) } & 15\end{array}$

$\begin{array}{ll}3.6 .2 \text { Diagram (b) } & 16\end{array}$

$\begin{array}{lll}3.6 .3 & \text { Renormalization } & 17\end{array}$

4 Results 18

$\begin{array}{lll}4.1 & \text { Leading order axion-baryon coupling } & 18\end{array}$

4.2 Loop corrections and estimation of the NNLO LECs 20

$\begin{array}{llr}5 & \text { Summary } & 24\end{array}$

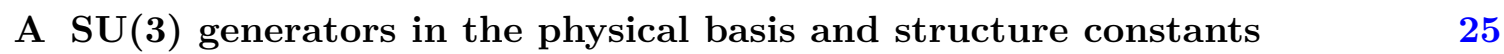

B Matrix elements of $g_{A B}, \hat{d}_{A B}$, and $g_{A B}^{\text {loop }} \quad 28$

\section{Introduction}

Soon after the discovery of the instanton solution of euclidean Yang-Mills gauge field theories [1], it has been realized that the vacuum structure of theories such as quantum chromodynamics (QCD) is highly non-trivial and that a so-called $\theta$-term appears [2]

$$
\mathcal{L}_{\mathrm{QCD}}=\mathcal{L}_{\mathrm{QCD}, 0}+\theta\left(\frac{g}{4 \pi}\right)^{2} \operatorname{Tr}\left[G_{\mu \nu} \tilde{G}^{\mu \nu}\right],
$$

which is $C P$ non-invariant as long as no quark is massless and as long as the effective angle $\bar{\theta}=\theta+\operatorname{Arg} \operatorname{det} \mathcal{M}$ is non-zero, where $\mathcal{M}$ refers to the quark mass matrix. In eq. (1.1), $\mathcal{L}_{\mathrm{QCD}, 0}$ denotes the usual QCD Lagrangian without the $\theta$-term, $g$ is the QCD coupling 
constant, $G_{\mu \nu}$ the gluon field strength tensor, $\tilde{G}^{\mu \nu}=\epsilon^{\mu \nu \alpha \beta} G_{\alpha \beta} / 2$ its dual, and Tr denotes the trace in color space. As a consequence, the neutron acquires an electric dipole moment $\propto \bar{\theta}[3]$. Theoretical estimations of the $\bar{\theta}$-induced neutron electric dipole moment (nEDM) roughly vary between $\left|d_{n}\right| \approx 10^{-16} \bar{\theta} e \mathrm{~cm}$ and $\left|d_{n}\right| \approx 10^{-15} \bar{\theta} e \mathrm{~cm}$; see refs. [4-7]. The extreme small upper limit for the physical value of the nEDM determined in experiments (the most recent result is $\left|d_{n}\right|<1.8 \times 10^{-26} e \mathrm{~cm}$ (90\% C.L.) [8]) implies

$$
\bar{\theta} \lesssim 10^{-11}
$$

which is a rather unnatural value for a quantity that in principle might take on values between 0 and $2 \pi$ (where $\theta=\pi$ is a special point [9]). Even though $\bar{\theta}$ of $\mathcal{O}(1)$ would alter nuclear physics and big bang and stellar nucleosynthesis considerably [10], such anthropic considerations do not constrain $\bar{\theta}$ to such a tiny value. One possible way to explain why $\bar{\theta} \approx 0$ (in other words, to resolve the so-called "strong $C P$-problem") is the Peccei-Quinn mechanism [11, 12], which features a new global chiral symmetry, now usually labeled $\mathrm{U}(1)_{\mathrm{PQ}}$, and which automatically leads to $\bar{\theta}=0$. The physical relic of this mechanism is not only a $C P$-conserving QCD Lagrangian, but also a new, very light pseudoscalar pseudo-Nambu-Goldstone boson with zero bare mass called axion [13, 14], which soon after its theoretical description also became a serious dark matter candidate [15-21], possibly forming Bose-Einstein condensates [22]. Both, the fact that the Peccei-Quinn mechanism provides an elegant solution to the strong $C P$-problem, and that it at the same time might provide a solution to the missing matter problem of the universe, accounts for the unwavering interest in the axion, even though it has not been detected yet and its existence hence remains hypothetical.

The original ("visible") Peccei-Quinn-Weinberg-Wilczek (PQWW) axion with a decay constant at the electroweak scale and a mass in the $\mathrm{keV} / \mathrm{MeV}$ region seems to be ruled out experimentally [4, 23-25], although there are still attempts to make the experimental data compatible with the original model [26, 27]. However, these models require a lot of additional assumptions including that these axions restrictively couple to the first generation fermions (up and down quark, electron) and are accidentally pion-phobic. The clearly preferred models are the so-called "invisible" axion models such as the Kim-Shifman-VainsteinZakharov (KSVZ) axion model [28, 29] (which sometimes is called "hadronic" as it does not couple to leptons) or the Dine-Fischler-Srednicki-Zhitnitsky (DFSZ) axion model [30, 31]. Phenomenologically, the physical properties of such an (almost) invisible axion such as its mass and the couplings to standard model particles (quarks, leptons, gauge bosons) are governed by its expectedly large decay constant, which is traditionally estimated as being $[4,15,16,32]$

$$
10^{9} \mathrm{GeV} \lesssim f_{a} \lesssim 10^{12} \mathrm{GeV}
$$

such that the axion mass $[33,34]$

$$
m_{a} \approx 5.7\left(\frac{10^{12} \mathrm{GeV}}{f_{a}}\right) \times 10^{-6} \mathrm{eV}
$$


presumably would be somewhere between a few $\mu \mathrm{eV}$ and $0.1 \mathrm{eV}$. The lifetime of the QCD axion, which may decay into two photons, is incredibly large [33]

$$
\tau_{a} \approx\left(\frac{1 \mathrm{eV}}{m_{a}}\right)^{5} \times 10^{24} \mathrm{~s} .
$$

Note that the traditional axion window, eq. (1.3), is set in order to match cold dark matter requirements of the canonical invisible axion models that solve the strong $C P$ problem. However, axions and axion-like particles have been considered also in the context of several other models with decay constants considerably smaller or larger than the window of eq. (1.3). For example, in the case of hadronic axions a decay constant as small as $f_{a} \approx 10^{6} \mathrm{GeV}$ has been proposed [35], while in string theories axions and axion-like particles might appear at the GUT or even the Planck scale, i.e. $10^{15} \mathrm{GeV} \lesssim f_{a} \lesssim 10^{18} \mathrm{GeV}$ [36].

As a consequence of its (model-dependent) coupling to standard model particles, the axion of course also couples to composite particles such as mesons or baryons. One possible method of estimating constraints on the axion decay constant (or equivalently its mass) rests upon this coupling to baryons, in particular to nucleons, namely through nuclear bremsstrahlung processes in stellar objects [37-51] (see also the overviews [4, 33, 52, 53]). The leading order $a N$ coupling has been derived several times since the early days [23, 35, 54-56]. More recently, this leading order coupling has been reexamined in ref. [57]. Moreover, such an analysis has been carried out up-to-and-including $\mathcal{O}\left(p^{3}\right)$ in $\mathrm{SU}(2)$ heavy baryon chiral perturbation theory (HBCHPT) in our previous work [58], where $p$ denotes a small parameter (see below in section 2.2).

In this paper, we extend these previous studies using SU(3) HBCHPT in two regards: (i) we extend the calculations of the axion-nucleon couplings to the SU(3) case, up-to-andincluding $\mathcal{O}\left(p^{3}\right)$, and (ii) we derive the couplings of the axion to the full ground state baryon octet (section 4). At this point one might wonder whether this extension is indeed useful given the fact that the most relevant coupling of the axion to baryons, i.e. the coupling to nucleons, is already known to good precision in the literature cited above, certainly precise enough for any phenomenological purpose. In this sense, the present study on the one hand is of rather theoretical interest, unraveling the explicit NNLO structure of the axion-nucleon coupling explicitly respecting the strange quark mass dependence. On the other hand, however, extending the analysis to the SU(3) case, we show that the traditional "invisible" axion also significantly couples to hyperons which has further phenomenological implications, since it has been suggested that hyperons might exist in the cores of neutron stars [59-86]. While it is a matter of ongoing research whether the seemingly energetically inevitable existence of hyperons in dense cores are compatible with the observed maximum masses of neutron stars (this question is related to the expected softening of the equation of state and known in the literature as "hyperon puzzle"), it is clear that approaches to constrain axion properties from cooling of neutron stars based on axion-nucleon bremsstrahlung alone might turn out to be insufficient. Depending on the underlying model and parameter sets, particularly the $\Lambda$ and the $\Sigma^{-}$might appear in significant fractions of the total baryon number. As will be shown in this study, the axion coupling to these particular hyperons is of a similar order as that to the nucleons (in fact, 
it could even be much larger than that to the neutron depending on the axion models), suggesting a revision of axion parameter constraints from stellar cooling.

Before performing the calculations of the axion-baryon couplings, we start with the explicit implementation of our framework and work out its ingredients and building blocks, which are the main topics of the following section 2. This section ends with some remarks of the general form of the axion-baryon coupling (section 2.3). The actual calculations of the axion-baryon couplings up to the leading one-loop level are performed in section 3 , and the results are discussed in section 4.

\section{$2 \mathrm{SU}(3)$ chiral perturbation theory with axions: general remarks}

\subsection{Ingredients from the axion-quark interaction Lagrangian}

Consider the general QCD Lagrangian with axions below the electroweak symmetry breaking scale [32]

$$
\mathcal{L}_{\mathrm{QCD}}=\mathcal{L}_{\mathrm{QCD}, 0}+\frac{a}{f_{a}}\left(\frac{g}{4 \pi}\right)^{2} \operatorname{Tr}\left[G_{\mu \nu} \tilde{G}^{\mu \nu}\right]+\bar{q} \gamma^{\mu} \gamma_{5} \frac{\partial_{\mu} a}{2 f_{a}} \mathcal{X}_{q} q
$$

where $q=(u, d, s, c, b, t)^{\mathrm{T}}$ collects the quark fields and $a$ refers to the axion field with decay constant $f_{a}$. The second term is a remnant of the $\theta$-term of eq. (1.1) after the spontaneous breakdown of the Peccei-Quinn symmetry. Depending on the underlying axion model, axions might additionally couple directly to the quark fields, which is here present in the form of the last term. Here, we assume the canonical scenario that the couplings are flavor conserving at tree-level, i.e. $\mathcal{X}_{q}=\operatorname{diag}\left\{X_{q}\right\}$ is a diagonal $6 \times 6$ matrix acting in flavor space, where the $X_{q}$ 's, $q=\{u, d, s, c, b, t\}$, are the coupling constants of the respective axion-quark interactions. These are given, for instance, by

$$
\begin{aligned}
X_{q}^{\mathrm{KSVZ}} & =0, \\
X_{u, c, t}^{\mathrm{DFSZ}} & =\frac{1}{3} \frac{x^{-1}}{x+x^{-1}}=\frac{1}{3} \sin ^{2} \beta, \\
X_{d, s, b}^{\mathrm{DFSZ}} & =\frac{1}{3} \frac{x}{x+x^{-1}}=\frac{1}{3} \cos ^{2} \beta=\frac{1}{3}-X_{u, c, t}^{\mathrm{DFSZ}},
\end{aligned}
$$

for the KSVZ-type axions and DFSZ-type axions, respectively, where $x=\cot \beta$ is the ratio of the vacuum expectation values (VEVs) of the two Higgs doublets within the latter models. Note that $\mathcal{L}_{\mathrm{QCD}, 0}$ in eq. (2.1) also contains a quark mass term $\bar{q} \mathcal{M}_{q} q$, with $\mathcal{M}_{q}=$ diag $\left\{m_{q}\right\}$ being the real, diagonal and $\gamma_{5}$-free quark mass matrix.

As usual, it is advisable to perform a transformation on the quark fields,

$$
q \rightarrow \exp \left(i \gamma_{5} \frac{a}{2 f_{a}} \mathcal{Q}_{a}\right) q
$$

in order to remove the second term of eq. (2.1). Choosing

$$
\mathcal{Q}_{a}=\frac{\mathcal{M}_{q}^{-1}}{\left\langle\mathcal{M}_{q}^{-1}\right\rangle} \approx \frac{1}{1+z+w} \operatorname{diag}(1, z, w, 0,0,0),
$$


which corresponds to vacuum alignment in the $\theta$-vacuum case, we can avoid the leading order mass mixing between the axion and the neutral pseudo-Nambu-Goldstone bosons of the spontaneous breaking of SU(3) chiral symmetry, the $\pi^{0}$ and the $\eta$, from the beginning. Here, $\langle\ldots\rangle$ denotes the trace in flavor space, $z=m_{u} / m_{d}$ and $w=m_{u} / m_{s}$. Now the axion-quark interaction Lagrangian is given by

$$
\mathcal{L}_{a-q}=-\left(\bar{q}_{L} \mathcal{M}_{a} q_{R}+\text { h.c. }\right)+\bar{q} \gamma^{\mu} \gamma_{5} \frac{\partial_{\mu} a}{2 f_{a}}\left(\mathcal{X}_{q}-\mathcal{Q}_{a}\right) q,
$$

where $q_{L}$ and $q_{R}$ are the left- and right-handed projections of the quark fields, and

$$
\mathcal{M}_{a}=\exp \left(i \frac{a}{f_{a}} \mathcal{Q}_{a}\right) \mathcal{M}_{q}
$$

Considering only the three-dimensional subspace of flavor space, i.e. $q=(u, d, s)^{\mathrm{T}}$, and introducing

$$
\begin{aligned}
& c^{(1)}=\frac{1}{3}\left(X_{u}+X_{d}+X_{s}-1\right), \\
& c^{(3)}=\frac{1}{2}\left(X_{u}-X_{d}-\frac{1-z}{1+z+w}\right), \\
& c^{(8)}=\frac{1}{2 \sqrt{3}}\left(X_{u}+X_{d}-2 X_{s}-\frac{1+z-2 w}{1+z+w}\right),
\end{aligned}
$$

we can decompose the matrix $\mathcal{X}_{q}-\mathcal{Q}_{a}$ into traceless parts and parts with non-vanishing trace, so that

$$
\begin{aligned}
\mathcal{L}_{a-q}= & -\left(\bar{q}_{L} \mathcal{M}_{a} q_{R}+\text { h.c. }\right) \\
& +\left(\bar{q} \gamma^{\mu} \gamma_{5} \frac{\partial_{\mu} a}{2 f_{a}}\left(c^{(1)} \mathbb{1}+c^{(3)} \lambda_{3}+c^{(8)} \lambda_{8}\right) q\right)_{q=(u, d, s)^{\mathrm{T}}} \\
& +\sum_{q=\{c, b, t\}}\left(\bar{q} \gamma^{\mu} \gamma_{5} \frac{\partial_{\mu} a}{2 f_{a}} X_{q} q\right),
\end{aligned}
$$

where $\lambda_{3}$ and $\lambda_{8}$ refer to the third and eighth Gell-Mann matrices, respectively. From this form of the axion-quark interaction Lagrangian, we can directly read off the required ingredients for the axionic SU(3) heavy baryon chiral Lagrangian, i.e. the external currents

$$
\begin{aligned}
s & =\mathcal{M}_{a}, \\
a_{\mu} & =\frac{\partial_{\mu} a}{2 f_{a}}\left(c^{(3)} \lambda_{3}+c^{(8)} \lambda_{8}\right), \\
a_{\mu, i}^{(s)} & =c_{i} \frac{\partial_{\mu} a}{2 f_{a}} \mathbb{1}, \quad i=1, \ldots, 4 .
\end{aligned}
$$

Here we have set

$$
c_{1}=c^{(1)}, \quad c_{2}=X_{c}, \quad c_{3}=X_{b}, \quad c_{4}=X_{t} .
$$


Note that in contrast to usual chiral perturbation theory, it is also necessary to add isosinglet axial-vector currents $a_{\mu, i}^{(s)}$ in order to preserve the full QCD axion interaction. This is possible here, as the subtleties that usually arise due to the $\mathrm{U}(1)_{A}$ anomaly are absent, because the model is now anomaly-free.

If one considers also flavor-changing axion-quark couplings at tree-level, $\mathcal{X}_{q}$ would be non-diagonal. In this case, it is likewise appropriate to decompose $\mathcal{X}_{q}-\mathcal{Q}_{a}$ into traceless parts and parts with non-vanishing trace, such that

$$
a_{\mu}=\frac{\partial_{\mu} a}{2 f_{a}} \sum_{i=1}^{8} C^{(i)} \lambda_{i}
$$

with $C^{(i)}$ depending on the allowed flavor-changing processes and $\lambda_{i}$ the eight Gell-Mann matrices. This then would cause baryon conversion processes. At this point we note that depending on the underlying model Higgs loops may induce off-diagonal couplings, which would be loop-suppressed but might be relevant due to unsuppressed top Yukawa couplings [87]. As stated above, we here stick to the most prevalent models excluding such flavor-changing axion-quark couplings at tree-level.

\subsection{Building blocks of the chiral Lagrangian}

The framework we use in this paper is chiral perturbation theory (CHPT) including baryons, meaning the effective field theory of QCD in the low-energy sector. CHPT is used to explore meson-baryon systems based on a systematic expansion in small momenta and quark masses below the scale of the spontaneous breakdown of chiral symmetry, $\Lambda_{\chi} \sim 1 \mathrm{GeV}$, as first developed by Gasser, Sainio, and Švarc for the two-flavor case [88] and by Krause for the three-flavor case [89]. However, while baryons can easily be incorporated into CHPT in a consistent manner respecting all symmetries, the power-counting scheme engineered to systematically arrange the infinitely many terms allowed by the fundamental symmetries is spoiled by the fact that the baryon masses $m_{B}$, which do not vanish in the chiral limit, are roughly of the same order as $\Lambda_{\chi}$. One way to overcome this problem is to realize that all mass scales need to be assigned to a scaling in the power counting and thus to treat the baryons as extremely heavy, static fermions, which is the idea behind HBCHPT [90-93]. In this scheme, the four momentum of a baryon field is decomposed as

$$
k_{\mu}=m_{B} v_{\mu}+p_{\mu}
$$

with the four-velocity $v_{\mu}$ subject to the constraint $v^{2}=1$, and $p_{\mu}$ a small residual momentum satisfying $(v \cdot p) \ll m_{B}$. After integrating out the heavy degrees of freedom, the resulting Lagrangian is expressed in terms of the heavy baryon field $B$, which is now characterized by a fixed velocity $v_{\mu}$ (for convenience, we refrain from writing $B_{v}$ to explicitly mark the $v$ dependence). Additionally, it is possible to simplify the Dirac algebra by expressing any Dirac bilinear by means of $v_{\mu}$ and the Pauli-Lubanski spin operator

$$
S_{\mu}=\frac{i}{2} \gamma_{5} \sigma_{\mu \nu} v^{\nu}
$$


which satisfies the relations

$$
\left\{S_{\mu}, S_{\nu}\right\}=\frac{1}{2}\left(v_{\mu} v_{\nu}-g_{\mu \nu}\right), \quad\left[S_{\mu}, S_{\nu}\right]=i \epsilon_{\mu \nu \rho \sigma} v^{\rho} S^{\sigma}, \quad(v \cdot S)=0, \quad S^{2}=\frac{1-d}{4},
$$

where the last one is valid in $d$ spacetime dimensions. In eq. (2.14) and in what follows, $[$,$] refers to the commutator and \{$,$\} to the anticommutator.$

Any term in the effective Lagrangian consists of a number of elements from a small set of basic building blocks, from which we will present those that are relevant for axionic $\mathrm{SU}(3) \mathrm{HBCHPT}$. In this three-flavor case, the ground state baryon octet consisting of the nucleons $p$ and $n$ and the hyperons $\Sigma, \Lambda$, and $\Xi$ are collected in a single $3 \times 3$ matrix

$$
B=\left(\begin{array}{ccc}
\frac{1}{\sqrt{2}} \Sigma_{3}+\frac{1}{\sqrt{6}} \Lambda_{8} & \Sigma^{+} & p \\
\Sigma^{-} & -\frac{1}{\sqrt{2}} \Sigma_{3}+\frac{1}{\sqrt{6}} \Lambda_{8} & n \\
\Xi^{-} & \Xi^{0} & -\frac{2}{\sqrt{6}} \Lambda_{8}
\end{array}\right),
$$

where

$$
\begin{aligned}
& \Sigma_{3}=\cos \epsilon \Sigma^{0}-\sin \epsilon \Lambda, \\
& \Lambda_{8}=\sin \epsilon \Sigma^{0}+\cos \epsilon \Lambda .
\end{aligned}
$$

Equation (2.15) corresponds to the adjoint representation of SU(3). Due to isospin breaking, the physical $\Sigma^{0}$ and $\Lambda$ are mixed states made of the $\Sigma_{3}$ and $\Lambda_{8}$. This mixing is usually parameterized by the mixing angle $\epsilon$ and one has

$$
\tan 2 \epsilon=\frac{\left\langle\lambda_{3} \mathcal{M}_{q}\right\rangle}{\left\langle\lambda_{8} \mathcal{M}_{q}\right\rangle}
$$

The pseudoscalar mesons, the pseudo-Nambu-Goldstone bosons of the spontaneous breakdown of chiral symmetry, appear in the Lagrangian in form of a unitary matrix

$$
u=\sqrt{U}=\exp \left(i \frac{\Phi}{2 F_{p}}\right)
$$

where $F_{p}$ is the pseudoscalar decay constant in the chiral limit and $\Phi$ is a Hermitian $3 \times 3$ matrix given by

$$
\Phi=\sqrt{2}\left(\begin{array}{ccc}
\frac{1}{\sqrt{2}} \pi_{3}+\frac{1}{\sqrt{6}} \eta_{8} & \pi^{+} & K^{+} \\
\pi^{-} & -\frac{1}{\sqrt{2}} \pi_{3}+\frac{1}{\sqrt{6}} \eta_{8} & K^{0} \\
K^{-} & \bar{K}^{0} & -\frac{2}{\sqrt{6}} \eta_{8}
\end{array}\right) .
$$

Again, the physical mass eigenstates of the neutral particles of the diagonal are mixed states as a consequence of isospin breaking effects and one has

$$
\begin{aligned}
& \pi_{3}=\cos \epsilon \pi^{0}-\sin \epsilon \eta, \\
& \eta_{8}=\sin \epsilon \pi^{0}+\cos \epsilon \eta .
\end{aligned}
$$


Using the leading order meson masses,

$$
\begin{aligned}
M_{\pi^{ \pm}}^{2} & =B_{0}\left(m_{u}+m_{d}\right), \\
M_{\pi^{0}}^{2} & =B_{0}\left(m_{u}+m_{d}\right)+\frac{2}{3} B_{0}\left(m_{u}+m_{d}-2 m_{s}\right) \frac{\sin ^{2} \epsilon}{\cos 2 \epsilon}, \\
M_{K^{ \pm}}^{2} & =B_{0}\left(m_{u}+m_{s}\right), \\
M_{K^{0}}^{2} & =B_{0}\left(m_{d}+m_{s}\right), \\
M_{\eta}^{2} & =\frac{1}{3} B_{0}\left(m_{u}+m_{d}+4 m_{s}\right)-\frac{2}{3} B_{0}\left(m_{u}+m_{d}-2 m_{s}\right) \frac{\sin ^{2} \epsilon}{\cos 2 \epsilon},
\end{aligned}
$$

where $B_{0}$ is a parameter from the $\mathcal{O}\left(p^{2}\right)$ meson Lagrangian related to the scalar quark condensate. One can further derive

$$
\begin{aligned}
\sin 2 \epsilon & =\frac{2}{\sqrt{3}} \frac{M_{K^{ \pm}}^{2}-M_{K^{0}}^{2}}{M_{\pi^{0}}^{2}-M_{\eta}^{2}}, \\
\cos 2 \epsilon & =\frac{2}{3} \frac{2 M_{\pi^{ \pm}}^{2}-M_{K^{ \pm}}^{2}-M_{K^{0}}^{2}}{M_{\pi^{0}}^{2}-M_{\eta}^{2}},
\end{aligned}
$$

using eq. (2.17), which is valid at leading order.

Any other particle such as the axion enters the theory in form of an external current. In the present case, we need five axial-vector currents $a_{\mu}$ and $a_{\mu, i}^{(s)}, i=1, \ldots, 4$, corresponding to the isovector and isoscalar parts of the axion-matter interaction; compare eq. (2.9). Furthermore, a scalar external field $s$ is needed to account for the explicit chiral symmetry breaking due to the light quark masses. Setting $\chi=2 B_{0} s$, it is then convenient to define

$$
\begin{aligned}
u_{\mu} & =i\left[u^{\dagger} \partial_{\mu} u-u \partial_{\mu} u^{\dagger}-i u^{\dagger} a_{\mu} u-i u a_{\mu} u^{\dagger}\right] \\
u_{\mu, i} & =i\left[-i u^{\dagger} a_{\mu, i}^{(s)} u-i u a_{\mu, i}^{(s)} u^{\dagger}\right]=2 a_{\mu, i}^{(s)} \\
\chi_{ \pm} & =u^{\dagger} \chi u^{\dagger} \pm u \chi^{\dagger} u
\end{aligned}
$$

which all transform in the same way under chiral transformations. Additionally, one defines the chiral covariant derivative

$$
\left[\mathcal{D}_{\mu}, B\right]=\partial_{\mu} B+\left[\Gamma_{\mu}, B\right]
$$

where

$$
\Gamma_{\mu}=\frac{1}{2}\left[u^{\dagger} \partial_{\mu} u+u \partial_{\mu} u^{\dagger}-i u^{\dagger} a_{\mu} u+i u a_{\mu} u^{\dagger}\right]
$$

is the chiral connection. Assigning systematically a chiral dimension $p$ to these building blocks in order to establish the power-counting method mentioned above, the effective Lagrangian is constructed by considering all combinations allowed by the underlying symmetries. The terms of the meson-baryon Lagrangian then can be arranged according to the chiral dimension,

$$
\mathcal{L}_{\Phi B}=\mathcal{L}_{\Phi B}^{(1)}+\mathcal{L}_{\Phi B}^{(2)}+\mathcal{L}_{\Phi B}^{(3)}+\cdots+\mathcal{L}_{\Phi}^{(2)}+\mathcal{L}_{\Phi}^{(4)}+\ldots
$$


where the superscript " $(i)$ " denotes the Lagrangian containing all terms of $\mathcal{O}\left(p^{i}\right)$. In the case of the purely mesonic Lagrangians $\mathcal{L}_{\Phi}^{(i)}, i$ is restricted to even numbers. Chiral loops start contributing at $\mathcal{O}\left(p^{3}\right)$ (see section 3.6), which means that $\mathcal{L}_{\Phi B}^{(3)}$ contains a number of low-energy constants and counter-terms needed for the renormalization (ch. 3.4 and 3.5). Note that in non-relativistic HBCHPT the Lagrangian (2.26) contains an inverse power series in $m_{B}$, the average octet mass in the chiral limit, which starts contributing at $\mathcal{O}\left(p^{2}\right)$ (see section 3.2). The leading order meson-baryon Lagrangian is given by

$$
\mathcal{L}_{\Phi B}^{(1)}=\left\langle i \bar{B} v^{\mu}\left[\mathcal{D}_{\mu}, B\right]\right\rangle+D\left\langle\bar{B} S^{\mu}\left\{u_{\mu}, B\right\}\right\rangle+F\left\langle\bar{B} S^{\mu}\left[u_{\mu}, B\right]\right\rangle+D^{i}\left\langle\bar{B} S^{\mu} u_{\mu, i} B\right\rangle,
$$

which in contrast to the two-flavor case contains two isovector axial-vector coupling constants $D$ and $F$, and in the present case of axionic HBCHPT the coupling constants $D^{i}$ to account for the isoscalar interactions.

\subsection{General form of the axion-baryon coupling}

For any process $B_{B} \rightarrow B_{A}+a$, where $B_{A}$ and $B_{B}$ denote arbitrary baryons and $a$ an axion, the general Feynman rule for the vertex in the baryon rest frame has the form

$$
\oint_{B_{B}}^{B_{A}} a=G_{a A B}(S \cdot q),
$$

where $q_{\mu}$ is the four-momentum of the outgoing axion. The coupling constant $G_{a A B}$ consists of a power series in $1 / f_{a}$

$$
G_{a A B}=-\frac{1}{f_{a}} g_{a A B}+\mathcal{O}\left(\frac{1}{f_{a}^{2}}\right) .
$$

Due to the expected huge value of $f_{a}$, it is sufficient to determine the leading order term $\propto g_{a A B} / f_{a}$, where

$$
g_{a A B}=g_{a A B}^{(1)}+g_{a A B}^{(2)}+g_{a A B}^{(3)}+\ldots
$$

can be expanded according to the power counting rules of HBCHPT. Here in particular

$$
\begin{aligned}
& g_{a A B}^{(1)}=g_{a A B}^{\mathrm{LO}, \text { tree }} \\
& g_{a A B}^{(2)}=g_{a A B}^{1 / m_{B}} \\
& g_{a A B}^{(3)}=g_{a A B}^{\mathrm{N}^{2} \mathrm{LO}, \text { tree }}+g_{a A B}^{1 / m_{B}^{2}}+g_{a A B}^{\mathrm{LO}, \text { loop }},
\end{aligned}
$$

where $g_{a A B}^{\mathrm{LO}}$ and $g_{a A B}^{\mathrm{N}^{2} \mathrm{LO}}$ refer to the contributions from the leading and next-to-next-toleading order chiral Lagrangian, i.e. from $\mathcal{L}_{\Phi B}^{(1)}$ and $\mathcal{L}_{\Phi B}^{(3)}$ of eq. (2.26), respectively. Furthermore, $g_{a A B}^{1 / m_{B}}$ and $g_{a A B}^{1 / m_{B}^{2}}$ refer to the $\mathcal{O}\left(m_{B}^{-1}\right)$ and $\mathcal{O}\left(m_{B}^{-2}\right)$ contributions from the expansion in the average baryon mass in the chiral limit $m_{B}$, which additionally appear in HBCHPT (see section 3.2). In this paper, we will determine each of these contributions to $g_{a A B}$ up to $\mathcal{O}\left(p^{3}\right)$.

As we work in the physical basis, $A$ and $B$ in $G_{a A B}$ and $g_{a A B}$ can be understood as $\mathrm{SU}(3)$ indices running from 1 to 8 that directly relate to the physical baryon fields. $G_{a 44}$, for 
instance, thus gives the axion-proton coupling $G_{a p p}$, whereas $G_{a 66}$ gives the axion-neutron coupling $G_{a n n}$ (see appendix A). In this basis, the matrix of $G_{a A B}$ is mostly diagonal as we exclude flavor-changing axion-quark couplings, i.e. $G_{a A B}=0$ for $A \neq B$, with the only exception of $G_{a 38}=G_{a 83}$, i.e. $G_{a \Sigma^{0} \Lambda}$, which is related to the $\Sigma^{0}-\Lambda$ mixing.

\section{Determining the axion-baryon coupling}

\subsection{Leading order: tree level contributions}

From the Lagrangian eq. (2.27), we can determine the leading order axion-baryon coupling by inserting ${ }^{1}$

$$
\begin{aligned}
\Gamma_{\mu} & =0 \\
u_{\mu} & =\frac{\partial_{\mu} a}{f_{a}}\left(c^{(3)} \lambda_{3}+c^{(8)} \lambda_{8}\right), \\
u_{\mu, i} & =c_{i} \frac{\partial_{\mu} a}{f_{a}} \mathbb{1},
\end{aligned}
$$

which gives

$$
\mathcal{L}_{\Phi B}^{(1), \text { int }}=\frac{g_{A B}}{f_{a}} \bar{B}_{A}(S \cdot \partial a) B_{B},
$$

where $A$ and $B$ are $\mathrm{SU}(3)$ indices in the physical basis (see appendix A). Here we have defined

$$
\begin{aligned}
g_{A B}=\frac{1}{2}\{D & \left(c^{(3)}\left\langle\tilde{\lambda}_{A}^{\dagger}\left\{\lambda_{3}, \tilde{\lambda}_{B}\right\}\right\rangle+c^{(8)}\left\langle\tilde{\lambda}_{A}^{\dagger}\left\{\lambda_{8}, \tilde{\lambda}_{B}\right\}\right\rangle\right) \\
& \left.+F\left(c^{(3)}\left\langle\tilde{\lambda}_{A}^{\dagger}\left[\lambda_{3}, \tilde{\lambda}_{B}\right]\right\rangle+c^{(8)}\left\langle\tilde{\lambda}_{A}^{\dagger}\left[\lambda_{8}, \tilde{\lambda}_{B}\right]\right\rangle\right)+2 c_{i} D^{i} \delta_{A B}\right\} .
\end{aligned}
$$

The matrix elements of $g_{A B}$ are given in table 4 in appendix B. As we are working in the physical basis including the $\Sigma^{0}-\Lambda$ mixing, $g_{A B}$ actually includes effects of $\mathcal{O}\left(p^{2}\right)$. The pure leading order axion-baryon coupling is found by setting $\epsilon=0$, i.e. by replacing $\tilde{\lambda}_{A / B} \rightarrow$ $\hat{\lambda}_{A / B}$, see eq. (A.14) of appendix A. In this case, $\lambda_{3}$ and $\lambda_{8}$ can be replaced by $\hat{\lambda}_{3}$ and $\hat{\lambda}_{8}$ so that the coupling can be expressed by means of the structure constants $\hat{f}_{A B C}$ and $\hat{d}_{A B C}$ :

$$
g_{A B}^{0}=\left.g_{A B}\right|_{\epsilon=0}=c^{(3)}\left(D \hat{d}_{A B 3}-F \hat{f}_{A B 3}\right)+c^{(8)}\left(D \hat{d}_{A B 8}-F \hat{f}_{A B 8}\right)+c_{i} D^{i} \delta_{A B} .
$$

It is hence clear that $g_{a A B}^{(1)}$ in eq. (2.30) is simply given by

$$
g_{a A B}^{(1)}=g_{A B}^{0} .
$$

\footnotetext{
${ }^{1}$ Note that in our previous work [58], there is a typo in the corresponding $\mathrm{SU}(2)$ equations, i.e. eqs. (3.16) and (3.21): $\tilde{u}_{\mu, i}$ is not given by $\tilde{u}_{\mu, i}=c_{i} \frac{\partial_{\mu} a}{f_{a}} \tau_{3}$. The correct expression is $\tilde{u}_{\mu, i}=c_{i} \frac{\partial_{\mu} a}{f_{a}} \mathbb{1}$.
} 


\subsection{Expansion in the baryon mass}

The Lagrangian containing the corrections due to the finite baryon masses $m_{B}$ in the heavy-baryon expansion up to $\mathcal{O}\left(p^{3}\right)$ corresponding to terms proportional to $1 / m_{B}$ and $1 / m_{B}^{2}$ is given by [93]

$$
\mathcal{L}^{1 / m_{B}}=\bar{B}_{A}\left\{\frac{1}{2 m_{B}} \gamma_{0}\left[\mathcal{B}_{(1)}^{A C}\right]^{\dagger} \gamma_{0} \mathcal{B}_{(1)}^{C B}-\frac{1}{4 m_{B}^{2}} \gamma_{0}\left[\mathcal{B}_{(1)}^{A C}\right]^{\dagger} \gamma_{0} \mathcal{A}_{(1)}^{C D} \mathcal{B}_{(1)}^{D B}\right\} B_{B} .
$$

Here

$$
\begin{aligned}
\mathcal{A}_{(1)}^{A B}=\frac{1}{2}\left(\left\langle\tilde{\lambda}_{A}^{\dagger}\left[i(v \cdot \mathcal{D}), \tilde{\lambda}_{B}\right]\right\rangle\right. & +D\left\langle\tilde{\lambda}_{A}^{\dagger}\left\{(S \cdot u), \tilde{\lambda}_{B}\right\}\right\rangle \\
& \left.+F\left\langle\tilde{\lambda}_{A}^{\dagger}\left[(S \cdot u), \tilde{\lambda}_{B}\right]\right\rangle+D^{i}\left\langle\tilde{\lambda}_{A}^{\dagger}\left(S \cdot u_{i}\right) \tilde{\lambda}_{B}\right\rangle\right),
\end{aligned}
$$

and

$$
\begin{aligned}
\mathcal{B}_{(1)}^{A B}=\frac{1}{2}\left(\left\langle\tilde{\lambda}_{A}^{\dagger}\left[i \gamma^{\mu} \mathcal{D}_{\mu}^{\perp}, \tilde{\lambda}_{B}\right]\right\rangle\right. & -\frac{D}{2}\left\langle\tilde{\lambda}_{A}^{\dagger}\left\{(v \cdot u) \gamma_{5}, \tilde{\lambda}_{B}\right\}\right\rangle \\
& \left.-\frac{F}{2}\left\langle\tilde{\lambda}_{A}^{\dagger}\left[(v \cdot u) \gamma_{5}, \tilde{\lambda}_{B}\right]\right\rangle-\frac{D^{i}}{2}\left\langle\tilde{\lambda}_{A}^{\dagger}\left(v \cdot u_{i}\right) \gamma_{5} \tilde{\lambda}_{B}\right\rangle\right),
\end{aligned}
$$

where for any four-vector $x_{\mu}$

$$
x_{\mu}^{\perp}=v_{\mu}(v \cdot x)-x_{\mu} .
$$

In the present case of axionic HBCHPT, we find

$$
\begin{aligned}
& \mathcal{A}_{(1)}^{A B}=i(v \cdot \partial) \delta_{A B}+\frac{1}{f_{a}} g_{A B}(S \cdot \partial a), \\
& \mathcal{B}_{(1)}^{A B}=i \gamma^{\mu} \partial_{\mu}^{\perp} \delta_{A B}-\frac{1}{2 f_{a}} g_{A B}(v \cdot \partial a) \gamma_{5},
\end{aligned}
$$

with $g_{A B}$ as defined in eq. (3.3), so that

$$
\begin{aligned}
\mathcal{L}^{1 / m_{B}}=\frac{1}{f_{a}} \bar{B}_{A}\left\{\frac{i g_{A B}}{2 m_{B}}\{(S \cdot \partial),(v \cdot \partial a)\}+\frac{g_{A B}}{4 m_{B}^{2}}[\right. & -\partial^{\mu}(S \cdot \partial a) \partial_{\mu}+(v \cdot \partial)(S \cdot \partial a)(v \cdot \partial) \\
& -(\{(S \cdot \partial),(v \cdot \partial a)\}(v \cdot \partial)+\text { h.c. }) \\
& +((S \cdot \partial)(\partial a \cdot \partial)+\text { h.c. })]\} B_{B} \cdot(3.12)
\end{aligned}
$$

Let $p$ be the momentum of the incoming baryon, $p^{\prime}$ the momentum of the outgoing baryon, and $\omega^{(\prime)}=\left(v \cdot p^{(\prime)}\right)$, then the resulting vertex Feynman rule reads

$$
\begin{aligned}
\oint_{B_{B}}^{B_{A}} a= & -\frac{g_{A B}}{f_{a}}\left\{\frac{1}{2 m_{B}}\left(\omega-\omega^{\prime}\right)-\frac{1}{4 m_{B}^{2}}\left(\omega^{2}-\omega^{\prime 2}+\omega \omega^{\prime}-p^{2}\right)\right\}(S \cdot q) \\
& +\frac{g_{A B}}{f_{a}}\left\{\frac{1}{m_{B}}\left(\omega-\omega^{\prime}\right)-\frac{1}{2 m_{B}^{2}}\left(\omega^{2}-\omega^{\prime 2}-\frac{1}{2}\left(p^{2}-p^{\prime 2}\right)\right)\right\}(S \cdot p),
\end{aligned}
$$


which is in complete analogy to the $\mathrm{SU}(2)$ case [58]. In the baryon rest frame with $p=0$, $\omega=0, v=(1,0,0,0)^{\mathrm{T}}$, and $\omega^{\prime}=\left(v \cdot p^{\prime}\right)=-(v \cdot q)=-q_{0} \ll m_{B}$, where $q_{0}$ is the relativistic energy of the outgoing axion, we finally find (see eqs. (2.30) and (2.31))

$$
g_{a A B}^{\mathrm{LO}, \text { tree }}+g_{a A B}^{1 / m_{B}}+g_{a A B}^{1 / m_{B}^{2}}=g_{A B}\left\{1+\frac{q_{0}}{2 m_{B}}+\frac{q_{0}^{2}}{4 m_{B}^{2}}\right\} .
$$

\subsection{Next-to-next-to-leading order: contributions from $\chi_{-}$}

At next-to-next-to-leading order, there are several contributions that have to be considered. We can differentiate terms with finite low-energy constants (LECs) that are $\propto \chi_{-}$, terms with LECs having finite and ultraviolet (UV) divergent pieces $\propto \chi_{+}$, and terms proportional some LECs that have no finite pieces serving as counter-terms to cancel UV divergences from the loop contributions. We start with the former.

Up to $\mathcal{O}\left(1 / f_{a}\right), \chi_{-}$, see eqs. (2.6) and (2.23), can be written as

$$
\chi_{-}=\frac{4 i M_{\pi^{ \pm}}^{2}}{f_{a}} \frac{z}{(1+z)^{2}}\left(1+\frac{w}{1+z}\right)^{-1} a \mathbb{1},
$$

where $M_{\pi^{ \pm}}^{2}$ is the leading order mass of the charged pions given in eq. (2.21). The contributing terms of $\mathcal{L}_{\Phi B}^{(3)}$ are (here and in the following section, we enumerate the LECs according to the list of terms in ref. [95])

$$
\begin{aligned}
\mathcal{L}^{\chi_{-}}= & -i d_{2}\left(\left\langle(\partial \bar{B} \cdot S) \chi_{-} B\right\rangle+\left\langle\bar{B} \chi_{-}(S \cdot \partial B)\right\rangle\right) \\
& -i d_{3}\left(\left\langle(\partial \bar{B} \cdot S)\left\langle\chi_{-}\right\rangle B\right\rangle+\left\langle\bar{B}\left\langle\chi_{-}\right\rangle(S \cdot \partial B)\right\rangle\right) .
\end{aligned}
$$

Inserting eq. (3.15) yields

$$
\oint_{B_{B}}^{B_{A}} \stackrel{a}{.-}=\frac{4 M_{\pi^{ \pm}}^{2}}{f_{a}} \frac{z}{(1+z)^{2}}\left(1+\frac{w}{1+z}\right)^{-1}\left(d_{2}+3 d_{3}\right) \delta_{A B}(S \cdot q)
$$

The reason for writing the coupling in this way is to match it to the corresponding terms in the $\mathrm{SU}(2)$ case, which are given by [58]

$$
\oint_{N}^{N} \cdot a=\frac{4 M_{\pi^{ \pm}}^{2}}{f_{a}} \frac{z}{(1+z)^{2}}\left(d_{18}+2 d_{19}\right)(S \cdot q)
$$

where $N$ is the nucleon field, and $d_{18}$ and $d_{19}$ are LECs from the next-to-next-to-leading order $\mathrm{SU}(2) \pi N$ Lagrangian [96]. Apart from a substitution of the LECs, the main difference between the $\mathrm{SU}(3)$ case, eq. (3.17), and the $\mathrm{SU}(2)$ case, eq. (3.18), is the explicit effect of the strange quark mass $m_{s}$ in the factor $(1+w /(1+z))^{-1}$, which reduces to unity at $m_{s} \rightarrow \infty$, i.e. $w \rightarrow 0$. In the $\mathrm{SU}(3)$ case, the finite value of $w$ accounts for a correction of about two percent. 


\subsection{Next-to-next-to-leading order: contributions from $\chi_{+}$}

From eqs. (2.6) and (2.23), one can readily determine that

$$
\chi_{+}=4 B_{0} \mathcal{M}_{q}+\mathcal{O}\left(f_{a}^{-2}\right) .
$$

The relevant terms from $\mathcal{L}_{\Phi B}^{(3)}$ are [95]

$$
\begin{aligned}
\mathcal{L}^{\chi_{+}}= & d_{41}(\lambda)\left(\left\langle\bar{B} S^{\mu}\left[u_{\mu},\left[\chi_{+}, B\right]\right]\right\rangle+\left\langle\bar{B} S^{\mu}\left[\chi_{+},\left[u_{\mu}, B\right]\right]\right\rangle\right) \\
& +d_{42}(\lambda)\left(\left\langle\bar{B} S^{\mu}\left[u_{\mu},\left\{\chi_{+}, B\right\}\right]\right\rangle+\left\langle\bar{B} S^{\mu}\left\{\chi_{+},\left[u_{\mu}, B\right]\right\}\right\rangle\right) \\
& +d_{43}(\lambda)\left(\left\langle\bar{B} S^{\mu}\left\{u_{\mu},\left[\chi_{+}, B\right]\right\}\right\rangle+\left\langle\bar{B} S^{\mu}\left[\chi_{+},\left\{u_{\mu}, B\right\}\right]\right\rangle\right) \\
& +d_{44}(\lambda)\left(\left\langle\bar{B} S^{\mu}\left\{u_{\mu},\left\{\chi_{+}, B\right\}\right\}\right\rangle+\left\langle\bar{B} S^{\mu}\left\{\chi_{+},\left\{u_{\mu}, B\right\}\right\}\right\rangle\right) \\
& +d_{45}(\lambda)\left\langle\bar{B} S^{\mu}\left[u_{\mu}, B\right]\right\rangle\left\langle\chi_{+}\right\rangle+d_{46}(\lambda)\left\langle\bar{B} S^{\mu}\left\{u_{\mu}, B\right\}\right\rangle\left\langle\chi_{+}\right\rangle \\
& +d_{47}(\lambda)\left\langle\bar{B} S^{\mu} B\right\rangle\left\langle u_{\mu} \chi_{+}\right\rangle+d_{43}^{i}(\lambda)\left\langle\bar{B}\left(S \cdot u_{i}\right)\left[\chi_{+}, B\right]\right\rangle \\
& +d_{44}^{i}(\lambda)\left\langle\bar{B}\left(S \cdot u_{i}\right)\left\{\chi_{+}, B\right\}\right\rangle+d_{46}^{i}(\lambda)\left\langle\bar{B}\left(S \cdot u_{i}\right) B\right\rangle\left\langle\chi_{+}\right\rangle .
\end{aligned}
$$

The LECs depend on the scale $\lambda$ and are given by

$$
d_{k}^{(i)}(\lambda)=d_{k}^{(i), r}(\lambda)+\frac{\beta_{k}^{(i)}}{F_{p}^{2}} L(\lambda), \quad k=\{41, \ldots, 47\} .
$$

In this equation, $d_{k}^{(i), r}(\lambda)$ refer to the renormalized LECs, and $L(\lambda)$ contains the pole for spacetime dimension $d=4$,

$$
L(\lambda)=\frac{\lambda^{d-4}}{(4 \pi)^{2}}\left(\frac{1}{d-4}-\frac{1}{2}[\ln (4 \pi)-\gamma+1]\right),
$$

where $\gamma$ is the Euler-Mascheroni constant. The $\beta$-functions are set to cancel the divergences of the one-loop functional, as discussed below. We write the resulting tree-level vertex as

$$
\int_{B_{B}}^{B_{A}} a=-\frac{4 M_{\pi^{ \pm}}^{2}}{f_{a}} \frac{z}{1+z} \hat{d}_{A B}(\lambda)(S \cdot q)
$$

where we have set

$$
\begin{aligned}
\hat{d}_{A B}(\lambda)=\frac{1}{2 m_{u}}\left(c^{(3)}\{\right. & d_{41}(\lambda)\left(\left\langle\hat{\lambda}_{A}^{\dagger}\left[\lambda_{3},\left[\mathcal{M}_{q}, \hat{\lambda}_{B}\right]\right]\right\rangle+\left\langle\hat{\lambda}_{A}^{\dagger}\left[\mathcal{M}_{q},\left[\lambda_{3}, \hat{\lambda}_{B}\right]\right]\right\rangle\right) \\
& +d_{42}(\lambda)\left(\left\langle\hat{\lambda}_{A}^{\dagger}\left[\lambda_{3},\left\{\mathcal{M}_{q}, \hat{\lambda}_{B}\right\}\right]\right\rangle+\left\langle\hat{\lambda}_{A}^{\dagger}\left\{\mathcal{M}_{q},\left[\lambda_{3}, \hat{\lambda}_{B}\right]\right\}\right\rangle\right) \\
& +d_{43}(\lambda)\left(\left\langle\hat{\lambda}_{A}^{\dagger}\left\{\lambda_{3},\left[\mathcal{M}_{q}, \hat{\lambda}_{B}\right]\right\}\right\rangle+\left\langle\hat{\lambda}_{A}^{\dagger}\left[\mathcal{M}_{q},\left\{\lambda_{3}, \hat{\lambda}_{B}\right\}\right]\right\rangle\right) \\
& +d_{44}(\lambda)\left(\left\langle\hat{\lambda}_{A}^{\dagger}\left\{\lambda_{3},\left\{\mathcal{M}_{q}, \hat{\lambda}_{B}\right\}\right\}\right\rangle+\left\langle\hat{\lambda}_{A}^{\dagger}\left\{\mathcal{M}_{q},\left\{\lambda_{3}, \hat{\lambda}_{B}\right\}\right\}\right\rangle\right)
\end{aligned}
$$




$$
\begin{aligned}
&+d_{45}(\lambda)\left\langle\hat{\lambda}_{A}^{\dagger}\left[\lambda_{3}, \hat{\lambda}_{B}\right]\right\rangle\left\langle\mathcal{M}_{q}\right\rangle+d_{46}(\lambda)\left\langle\hat{\lambda}_{A}^{\dagger}\left\{\lambda_{3}, \hat{\lambda}_{B}\right\}\right\rangle\left\langle\mathcal{M}_{q}\right\rangle \\
&\left.+2 d_{47}(\lambda) \delta_{A B}\left\langle\lambda_{3} \mathcal{M}_{q}\right\rangle\right\} \\
&+c^{(8)}\left\{d_{41}(\lambda)\left(\left\langle\hat{\lambda}_{A}^{\dagger}\left[\lambda_{8},\left[\mathcal{M}_{q}, \hat{\lambda}_{B}\right]\right]\right\rangle+\left\langle\hat{\lambda}_{A}^{\dagger}\left[\mathcal{M}_{q},\left[\lambda_{8}, \hat{\lambda}_{B}\right]\right]\right\rangle\right)\right. \\
&+d_{42}(\lambda)\left(\left\langle\hat{\lambda}_{A}^{\dagger}\left[\lambda_{8},\left\{\mathcal{M}_{q}, \hat{\lambda}_{B}\right\}\right]\right\rangle+\left\langle\hat{\lambda}_{A}^{\dagger}\left\{\mathcal{M}_{q},\left[\lambda_{8}, \hat{\lambda}_{B}\right]\right\}\right\rangle\right) \\
&+d_{43}(\lambda)\left(\left\langle\hat{\lambda}_{A}^{\dagger}\left\{\lambda_{8},\left[\mathcal{M}_{q}, \hat{\lambda}_{B}\right]\right\}\right\rangle+\left\langle\hat{\lambda}_{A}^{\dagger}\left[\mathcal{M}_{q},\left\{\lambda_{8}, \hat{\lambda}_{B}\right\}\right]\right\rangle\right) \\
&+d_{44}(\lambda)\left(\left\langle\hat{\lambda}_{A}^{\dagger}\left\{\lambda_{8},\left\{\mathcal{M}_{q}, \hat{\lambda}_{B}\right\}\right\}\right\rangle+\left\langle\hat{\lambda}_{A}^{\dagger}\left\{\mathcal{M}_{q},\left\{\lambda_{8}, \hat{\lambda}_{B}\right\}\right\}\right\rangle\right) \\
&+d_{45}(\lambda)\left\langle\hat{\lambda}_{A}^{\dagger}\left[\lambda_{8}, \hat{\lambda}_{B}\right]\right\rangle\left\langle\mathcal{M}_{q}\right\rangle+d_{46}(\lambda)\left\langle\hat{\lambda}_{A}^{\dagger}\left\{\lambda_{8}, \hat{\lambda}_{B}\right\}\right\rangle\left\langle\mathcal{M}_{q}\right\rangle \\
&\left.+2 d_{47}(\lambda) \delta_{A B}\left\langle\lambda_{8} \mathcal{M}_{q}\right\rangle\right\} \\
&+c_{i}\left\{d_{43}^{i}(\lambda)\left\langle\hat{\lambda}_{A}\left[\mathcal{M}_{q}, \hat{\lambda}_{B}\right]\right\rangle+d_{44}^{i}(\lambda)\left\langle\hat{\lambda}_{A}\left\{\mathcal{M}_{q}, \hat{\lambda}_{B}\right\}\right\rangle\right. \\
&\left.\left.+2 d_{46}^{i}(\lambda) \delta_{A B}\left\langle\mathcal{M}_{q}\right\rangle\right\}\right) .
\end{aligned}
$$

The matrix elements of $\hat{d}_{A B}$ are given in eqs. (B.1)-(B.9) of appendix B.

\subsection{Next-to-next-to-leading order: counter-terms}

The counter-terms needed for the renormalization have been worked out in ref. [93]. For the present case, we need the terms $i=\{36,37,38,39\}$ from this paper, which are given by

$$
\begin{aligned}
\mathcal{L}^{\text {c.t. }}= & d_{36}(\lambda)\langle(v \cdot \partial \bar{B})\{(S \cdot u),(v \cdot \partial B)\}\rangle \\
& +d_{37}(\lambda)\langle(v \cdot \partial \bar{B})[(S \cdot u),(v \cdot \partial B)]\rangle \\
& +d_{38}(\lambda)\langle\bar{B}\{[(v \cdot \partial),[(v \cdot \partial),(S \cdot u)]], B\}\rangle \\
& +d_{39}(\lambda)\langle\bar{B}[[(v \cdot \partial),[(v \cdot \partial),(S \cdot u)]], B]\rangle \\
& +d_{36}^{i}(\lambda)\left\langle(v \cdot \partial \bar{B})\left(S \cdot u_{i}\right)(v \cdot \partial B)\right\rangle \\
& +d_{38}^{i}(\lambda)\left\langle\bar{B}\left[(v \cdot \partial),\left[(v \cdot \partial),\left(S \cdot u_{i}\right)\right]\right] B\right\rangle,
\end{aligned}
$$

where we have added two terms in order to account for the isoscalar interactions. The LECs have no finite part, i.e.

$$
d_{k}^{(i)}(\lambda)=\frac{\beta_{k}^{(i)}}{F_{p}^{2}} L(\lambda), \quad k=\{36, \ldots, 39\} .
$$

Their contribution to the tree-level vertex is given by

$$
\oint_{B_{B}}^{B_{A}} a=-\frac{1}{f_{a}} \hat{d}_{A B}^{c . t}(\lambda)(S \cdot q)
$$




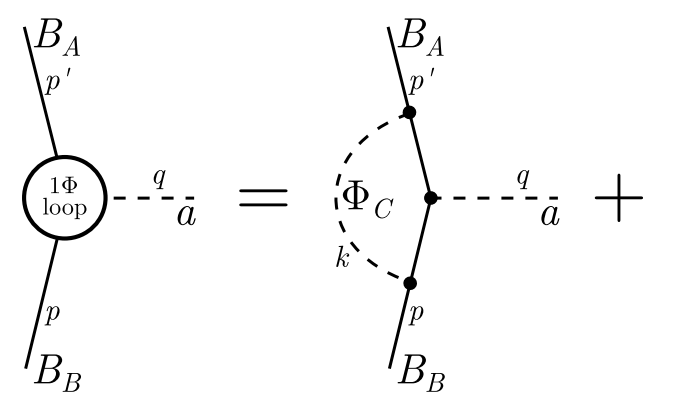

(a)

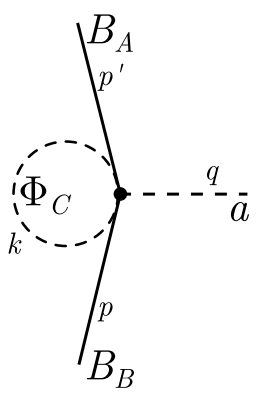

(b)

Figure 1. Non-vanishing meson loop contributions to $B_{B} \rightarrow B_{A}+a$.

with

$$
\begin{aligned}
\hat{d}_{A B}^{c . t .}(\lambda)= & \left(d_{36}(\lambda) \omega \omega^{\prime}-d_{38}(\lambda)\left(\omega-\omega^{\prime}\right)^{2}\right)\left(c^{(3)} \hat{d}_{A B 3}+c^{(8)} \hat{d}_{A B 8}\right) \\
& -\left(d_{37}(\lambda) \omega \omega^{\prime}-d_{39}(\lambda)\left(\omega-\omega^{\prime}\right)^{2}\right)\left(c^{(3)} \hat{f}_{A B 3}+c^{(8)} \hat{f}_{A B 8}\right) \\
& +\left(d_{36}^{i}(\lambda) \omega \omega^{\prime}-d_{38}^{i}(\lambda)\left(\omega-\omega^{\prime}\right)^{2}\right) c_{i} \delta_{A B} .
\end{aligned}
$$

\subsection{Loops}

There are only two non-vanishing single meson loops that contribute to the $\mathcal{O}\left(p^{3}\right)$ axionbaryon vertex at $\mathcal{O}\left(1 / f_{a}\right)$, which are shown in figure 1 . In this figure, mesons are identified by means of the $\mathrm{SU}(3)$ index $C$ in the physical basis including the $\pi^{0}-\eta$ mixing, cf. eqs. (2.19) and (A.3). Note that the potential diagrams with a $a \Phi B B$ vertex and a meson line connected to one baryon leg vanish because $(v \cdot S)=0$, see eq. (2.14), as has been shown in ref. [58] for the corresponding diagrams in the $\mathrm{SU}(2)$ case, which have the same topology.

\subsubsection{Diagram (a)}

Using the leading order meson-baryon vertex rule, one finds

$$
\begin{aligned}
(\mathrm{a})= & \frac{1}{f_{a} F_{p}^{2}} \sum_{C} g_{A B C}^{(a)} S^{\mu}(S \cdot q) S^{\nu} \frac{1}{i} \int \frac{\mathrm{d}^{d} k}{(2 \pi)^{d}} \frac{k_{\mu} k_{\nu}}{\left(k^{2}-M_{\Phi_{C}}^{2}+i \eta\right)\left(\omega^{\prime}-v \cdot k+i \eta\right)(\omega-v \cdot k+i \eta)} \\
= & \frac{1}{6 f_{a}} \frac{1}{\left(4 \pi F_{p}\right)^{2}} \sum_{C} g_{A B C}^{(a)}\left\{-M_{\Phi_{C}}^{2}\right. \\
& +\frac{1}{\omega-\omega^{\prime}}\left(\omega^{3}-\omega^{\prime 3}+2\left[\left(M_{\Phi_{C}}^{2}-\omega^{2}\right)^{\frac{3}{2}} \arccos \frac{-\omega}{M_{\Phi_{C}}}-\left(M_{\Phi_{C}}^{2}-\omega^{\prime 2}\right)^{\frac{3}{2}} \arccos \frac{-\omega^{\prime}}{M_{\Phi_{C}}}\right]\right) \\
& \left.+\left(3 M_{\Phi_{C}}^{2}-2\left(\omega-\omega^{\prime}\right)^{2}-6 \omega \omega^{\prime}\right)\left((4 \pi)^{2} L(\lambda)+\ln \frac{M_{\Phi_{C}}}{\lambda}\right)\right\}(S \cdot q),
\end{aligned}
$$


where we have applied dimensional regularization and used the properties in eq. (2.14). Moreover, we have defined

$$
\begin{aligned}
g_{A B C}^{(a)}=\frac{1}{4} \sum_{D, E}(D & \left.\left\langle\hat{\lambda}_{A}^{\dagger}\left\{\tilde{\lambda}_{C}, \hat{\lambda}_{E}\right\}\right\rangle+F\left\langle\hat{\lambda}_{A}^{\dagger}\left[\tilde{\lambda}_{C}, \hat{\lambda}_{E}\right]\right\rangle\right) g_{E D}^{0} \\
& \times\left(D\left\langle\hat{\lambda}_{D}^{\dagger}\left\{\tilde{\lambda}_{C}^{\dagger}, \hat{\lambda}_{B}\right\}\right\rangle+F\left\langle\hat{\lambda}_{D}^{\dagger}\left[\tilde{\lambda}_{C}^{\dagger}, \hat{\lambda}_{B}\right]\right\rangle\right),
\end{aligned}
$$

in order to handle all meson loops for all baryons $B_{A}$ and $B_{B}$ at the same time. Here $g_{E D}^{0}$ refers to eq. (3.4), the leading order axion-baryon coupling. Equation (3.29) contains UV divergences, which will be treated below in section 3.6.3. The expression for diagram (a) can be simplified by considering the baryon rest frame and expanding around $q_{0} \ll M_{\Phi_{C}}$ for all mesons $\Phi_{C}$, which yields

$$
\begin{aligned}
(\mathrm{a})= & \frac{1}{6 f_{a}} \sum_{C} g_{A B C}^{(a)}\left(\frac{M_{\Phi_{C}}}{4 \pi F_{p}}\right)^{2}\left\{1+\frac{3 \pi}{2} \frac{q_{0}}{M_{\Phi_{C}}}-\frac{5}{3}\left(\frac{q_{0}}{M_{\Phi_{C}}}\right)^{2}\right. \\
& \left.+\left(3-2\left(\frac{q_{0}}{M_{\Phi_{C}}}\right)^{2}\right)\left((4 \pi)^{2} L(\lambda)+\ln \frac{M_{\Phi_{C}}}{\lambda}\right)\right\}(S \cdot q) .
\end{aligned}
$$

\subsubsection{Diagram (b)}

Expanding $u$ up to order $\Phi^{2}$ yields

$$
u_{\mu}=-\frac{\partial_{\mu} a}{8 f_{a} F_{p}^{2}}\left(c^{(3)}\left[\Phi,\left[\Phi, \lambda_{3}\right]\right]+c^{(8)}\left[\Phi,\left[\Phi, \lambda_{8}\right]\right]\right)
$$

so the vertex rule for the $a B_{A} B_{B} \Phi_{C} \Phi_{C}$-vertex in the physical basis derived from $\mathcal{L}_{\Phi B}^{(1)}$ can be written as

$$
\frac{1}{f_{a} F_{p}^{2}} g_{A B C}^{(b)}(S \cdot q)
$$

where we have defined the coupling constant

$$
\begin{aligned}
g_{A B C}^{(b)}=\frac{1}{8}\left\{c^{(3)}\left(D\left\langle\hat{\lambda}_{A}^{\dagger}\left\{\left[\tilde{\lambda}_{C},\left[\tilde{\lambda}_{C}^{\dagger}, \lambda_{3}\right]\right], \hat{\lambda}_{B}\right\}\right\rangle+F\left\langle\hat{\lambda}_{A}^{\dagger}\left[\left[\tilde{\lambda}_{C},\left[\tilde{\lambda}_{C}^{\dagger}, \lambda_{3}\right]\right], \hat{\lambda}_{B}\right]\right\rangle\right)\right. \\
\left.+c^{(8)}\left(D\left\langle\hat{\lambda}_{A}^{\dagger}\left\{\left[\tilde{\lambda}_{C},\left[\tilde{\lambda}_{C}^{\dagger}, \lambda_{8}\right]\right], \hat{\lambda}_{B}\right\}\right\rangle+F\left\langle\hat{\lambda}_{A}^{\dagger}\left[\left[\tilde{\lambda}_{C},\left[\tilde{\lambda}_{C}^{\dagger}, \lambda_{8}\right]\right], \hat{\lambda}_{B}\right]\right\rangle\right)\right\} .
\end{aligned}
$$

In fact, this vertex and thus diagram (b) is independent of the mixing angle $\epsilon$ so one might as well substitute $\tilde{\lambda}_{C} \rightarrow \hat{\lambda}_{C}$ in eq. (3.34). Therefore, one can also express the coupling constant by means of the structure constants defined in eqs. (A.9) and (A.14),

$$
g_{A B C}^{(b)}=\frac{1}{4} \sum_{D, E} \hat{f}_{E C D}\left(c^{(3)} \hat{f}_{3 C D}\left[D \hat{d}_{A E B}+F \hat{f}_{A E B}\right]+c^{(8)} \hat{f}_{8 C D}\left[D \hat{d}_{A E B}+F \hat{f}_{A E B}\right]\right) .
$$


The loops of diagram (b) for all mesons $\Phi_{C}$ can then be calculated as

$$
\begin{aligned}
(\mathrm{b}) & =\frac{1}{2 f_{a} F_{p}^{2}}(S \cdot q) \sum_{C} g_{A B C}^{(b)} \frac{1}{i} \int \frac{\mathrm{d}^{d} k}{(2 \pi)^{d}} \frac{1}{k^{2}-M_{\Phi_{C}}^{2}+i \eta} \\
& =-\frac{1}{f_{a} F_{p}^{2}}(S \cdot q) \sum_{C} g_{A B C}^{(b)} M_{\Phi_{C}}^{2}\left(L(\lambda)+\frac{1}{(4 \pi)^{2}} \ln \frac{M_{\Phi_{c}}}{\lambda}\right) .
\end{aligned}
$$

\subsubsection{Renormalization}

The divergences appearing in the meson loop calculations in dimensional regularization are canceled by setting appropriate $\beta$ functions for the LECs appearing in $\hat{d}_{A B}(\lambda)$, eq. (3.24), and $\hat{d}_{A B}^{c . t}(\lambda)$, eq. $(3.27)$,

$$
\begin{array}{ll}
\beta_{36}=2 D\left(D^{2}+3 F^{2}\right), & \beta_{36}^{i}=-\frac{4}{3} D^{i}\left(13 D^{2}+9 F^{2}\right), \\
\beta_{37}=\frac{2}{3} F\left(5 D^{2}-9 F^{2}\right), & \beta_{38}=-\frac{2}{3} D\left(D^{2}+3 F^{2}\right), \\
\beta_{38}^{i}=\frac{4}{9} D^{i}\left(13 D^{2}+9 F^{2}\right), & \beta_{39}=-\frac{2}{9} F\left(5 D^{2}-9 F^{2}\right), \\
\beta_{41}=-\frac{1}{48} D\left(9 D^{2}+7 F^{2}-9\right), & \beta_{42}=-\frac{3}{16} F\left(D^{2}-F^{2}-1\right), \\
\beta_{43}=-\frac{1}{48} F\left(7 D^{2}+9 F^{2}-9\right), & \beta_{43}^{i}=-\frac{5}{6} D F D^{i}, \\
\beta_{44}=-\frac{1}{48} D\left(23 D^{2}+9 F^{2}-9\right), & \beta_{44}^{i}=-\frac{1}{4} D^{i}\left(D^{2}-3 F^{2}\right), \\
\beta_{45}=-\frac{1}{36} F\left(D^{2}-9 F^{2}-9\right), & \beta_{46}=\frac{1}{36} D\left(17 D^{2}-9 F^{2}+9\right), \\
\beta_{46}^{i}=\frac{1}{18} D^{i}\left(13 D^{2}+9 F^{2}\right) & \beta_{47}=\frac{1}{2} D\left(D^{2}+3 F^{2}-1\right),
\end{array}
$$

of which the $\beta_{k}$ 's are in accordance with the ones given in ref. [93], and the $\beta_{k}^{i}$, s have been worked out here for the first time. With that, the full renormalized $\mathcal{O}\left(p^{3}\right)$ contribution reads

$$
g_{a A B}^{\mathrm{N}^{2} \mathrm{LO}, \text { tree }}+g_{a A B}^{\mathrm{LO}, \text { loop }}=\frac{4 M_{\pi^{ \pm}}^{2} z}{1+z}\left(\hat{d}_{A B}^{r}(\lambda)-\frac{d_{2}+3 d_{3}}{1+z+w} \delta_{A B}\right)-\frac{1}{27} g_{A B}^{\text {loop }, r}+g_{A B}^{\text {loop }, \mathrm{sc}}(\lambda),
$$

where we have neglected terms of $\mathcal{O}\left(q_{0} / M_{\Phi_{C}}\right)$. Moreover, $\hat{d}_{A B}^{r}$ refers to eq. (3.24) with renormalized LECs, and

$$
\begin{aligned}
g_{A B}^{\text {loop }, r} & =\frac{9}{2} \sum_{C} g_{A B C}^{(a)}\left(\frac{M_{\Phi_{C}}}{4 \pi F_{p}}\right)^{2}, \\
g_{A B}^{\text {loop,sc }}(\lambda) & =\sum_{C}\left(-\frac{1}{2} g_{A B C}^{(a)}+g_{A B C}^{(b)}\right)\left(\frac{M_{\Phi_{C}}}{4 \pi F_{p}}\right)^{2} \ln \frac{M_{\Phi_{C}}}{\lambda} .
\end{aligned}
$$

The matrix elements of $g_{A B}^{\text {loop,r} r}$ are given in eqs. (B.10)-(B.18) of appendix B. 


\section{Results}

\subsection{Leading order axion-baryon coupling}

Using the nucleon matrix elements $\Delta q$ defined by $s^{\mu} \Delta q=\left\langle p\left|\bar{q} \gamma^{\mu} \gamma_{5} q\right| p\right\rangle, s^{\mu}$ being the spin of the proton, we set

$$
\begin{aligned}
(D+F) & =g_{A}=\Delta u-\Delta d, & -(D-3 F) & =\Delta u+\Delta d-2 \Delta s, \\
D^{1} & =\Delta u+\Delta d+\Delta s, & D^{i} & =\Delta q_{i}, \text { for } i=\{2,3,4\}, \Delta q_{i}=\{\Delta c, \Delta b, \Delta t\},
\end{aligned}
$$

where the respective baryon matrix elements are related to the ones from the nucleons by flavor symmetry. From now on, we neglect terms $\propto\{\Delta c, \Delta b, \Delta t\}$, which basically represent sea quark effects beyond the numerical uncertainties of the dominant contributions of the up, down, and strange quarks (at least in the standard DFSZ scenario, where the couplings to heavy quarks are of the same order as the couplings to the light quarks; if alternatively the couplings $X_{c}, X_{b}$, and $X_{t}$ or only one or two of them were much stronger than $X_{u}, X_{d}$, and $X_{s}$, these sea quark terms could not be ignored any longer). Inserting this into the result for the leading order axion-baryon coupling constant $g_{a A B}^{(1)}$, eq. (3.4) (see also table 4 in appendix B), we find

$$
\begin{aligned}
g_{a \Sigma^{+} \Sigma^{+}}^{(1)} & =-\frac{\Delta u+z \Delta s+w \Delta d}{1+z+w}+\Delta u X_{u}+\Delta s X_{d}+\Delta d X_{s}, \\
g_{a \Sigma^{-} \Sigma^{-}}^{(1)} & =-\frac{\Delta s+z \Delta u+w \Delta d}{1+z+w}+\Delta s X_{u}+\Delta u X_{d}+\Delta d X_{s}, \\
g_{a \Sigma^{0} \Sigma^{0}}^{(1)} & =-\frac{\frac{\Delta u+\Delta s}{2}(1+z)+w \Delta d}{1+z+w}+\frac{\Delta u+\Delta s}{2}\left(X_{u}+X_{d}\right)+\Delta d X_{s}, \\
g_{a p p}^{(1)}= & -\frac{\Delta u+z \Delta d+w \Delta s}{1+z+w}+\Delta u X_{u}+\Delta d X_{d}+\Delta s X_{s}, \\
g_{a \Xi^{-} \Xi^{-}}^{(1)}= & -\frac{\Delta s+z \Delta d+w \Delta u}{1+z+w}+\Delta s X_{u}+\Delta d X_{d}+\Delta u X_{s}, \\
g_{a n n}^{(1)}= & -\frac{\Delta d+z \Delta u+w \Delta s}{1+z+w}+\Delta d X_{u}+\Delta u X_{d}+\Delta s X_{s}, \\
g_{a \Xi^{0} \Xi^{0}}^{(1)}= & -\frac{\Delta d+z \Delta s+w \Delta u}{1+z+w}+\Delta d X_{u}+\Delta s X_{d}+\Delta u X_{s}, \\
g_{a \Lambda \Lambda}^{(1)}= & -\frac{\frac{\Delta u+4 \Delta d+\Delta s}{6}(1+z)+\frac{2 \Delta u-\Delta d+2 \Delta s}{3} w}{1+z+w} \\
g_{a \Sigma^{0} \Lambda}^{(1)}= & \frac{\frac{\Delta u-2 \Delta d+\Delta s}{2 \sqrt{3}}(1-z)}{1+z+w}+\frac{\Delta u-2 \Delta d+\Delta s}{2 \sqrt{3}}\left(X_{u}-X_{d}\right) . \\
+\frac{\Delta u}{6}+4 d+\Delta s & \left.X_{u}+X_{d}\right)+\frac{2 \Delta u-\Delta d+2 \Delta s}{3},
\end{aligned}
$$

In particular, $g_{a p p}^{(1)}$ and $g_{a n n}^{(1)}$ are exactly the same as in the $\mathrm{SU}(2)$ case. Using [97]

$$
\Delta u=0.847(50), \quad \Delta d=-0.407(34), \quad \Delta s=-0.035(13),
$$


which correspond to

$$
\begin{aligned}
D & =\frac{1}{2} \Delta u-\Delta d+\frac{1}{2} \Delta s=0.813(43), \\
F & =\frac{1}{2} \Delta u-\frac{1}{2} \Delta s=0.441(26), \\
D^{1} & =0.405(62)
\end{aligned}
$$

and $[97]$

$$
z=0.485(19), \quad w=0.025(1)
$$

we obtain

$$
\begin{aligned}
g_{a \Sigma^{+} \Sigma^{+}}^{(1)} & =-0.543(34)+0.847(50) X_{u}-0.035(13) X_{d}-0.407(34) X_{s}, \\
g_{a \Sigma^{-} \Sigma^{-}}^{(1)} & =-0.242(21)-0.035(13) X_{u}+0.847(50) X_{d}-0.407(34) X_{s}, \\
g_{a \Sigma^{0} \Sigma^{0}}^{(1)} & =-0.396(25)+0.417(25) X_{u}+0.395(25) X_{d}-0.407(35) X_{s}, \\
g_{a p p}^{(1)} & =-0.430(36)+0.847(50) X_{u}-0.407(34) X_{d}-0.035(13) X_{s}, \\
g_{a \Xi^{-} \Xi^{-}}^{(1)} & =0.140(15)-0.035(13) X_{u}-0.407(34) X_{d}+0.847(50) X_{s}, \\
g_{a n n}^{(1)} & =-0.002(30)-0.407(34) X_{u}+0.847(50) X_{d}-0.035(13) X_{s}, \\
g_{a \Xi^{0} \Xi^{0}}^{(1)} & =0.267(23)-0.407(34) X_{u}-0.035(13) X_{d}+0.847(50) X_{s}, \\
g_{a \Lambda \Lambda}^{(1)} & =0.126(25)-0.147(25) X_{u}-0.125(25) X_{d}+0.677(35) X_{s}, \\
g_{a \Sigma^{0} \Lambda}^{(1)} & =-0.153(10)+0.463(25) X_{u}-0.476(25) X_{d}+0.013(1) X_{s},
\end{aligned}
$$

where we also considered corrections from the non-vanishing mixing angle $\epsilon$ related to isospin breaking in the cases of the $\Sigma^{0}$ and the $\Lambda$ (which is why there also appears a term $\propto X_{s}$ in $g_{a \Sigma^{0} \Lambda}^{(1)}$. In table 1 , we list the results for the KSVZ axion, where $X_{q}=0$, and the DFSZ axion, where the axion-quark couplings $X_{q}$ depend on the angle $\beta$ related to the VEVs of the involved Higgs doublets (see above, eq. (2.2)). In the KSVZ model, the strongest couplings are hence to be expected for the $\Sigma^{+}$and the proton, which is also true for the DFSZ model at small values of $\sin ^{2} \beta$ (in this region, also the $\Xi^{0}$ shows a considerably large coupling with an opposite sign). As noted already in many previous works, the axionneutron coupling in some scenarios is strongly suppressed and might even vanish in the KSVZ model and the DFSZ model at $\sin ^{2} \beta \approx 2 / 3$ (corresponding to $x=1 / \sqrt{2}$, where $x$ is the ratio of the VEVs of the two Higgs doublets). The axion-neutron coupling is also the only baryon conserving coupling that might vanish in the DFSZ model, as it is the only one that changes its sign when varying $\sin ^{2} \beta$ from zero to unity. At $\sin ^{2} \beta=1$, also the $\Sigma^{0}-\Lambda$ mixing vertex disappears. At the same value of $\sin ^{2} \beta$, the couplings are somehow "harmonized", i.e. the couplings of the axion to particles of the same strangeness $S$ are approximately the same, which is due to flavor symmetry. In case of the neutron and the proton with $S=0$ one then has $g_{a A B}^{(1), \sin ^{2} \beta=1} \approx-0.14$, in case of the $\Sigma$ particles with $S=1$, one has $g_{a A B}^{(1), \sin ^{2} \beta=1} \approx-0.26$, and in case of the two $\Xi$ baryons with $S=2$, one has $g_{a A B}^{(1), \sin ^{2} \beta=1} \approx 0.13$. The difference among the particles with the same $S$ can be determined 


\begin{tabular}{|c|c|c|c|c|c|}
\hline \multirow{3}{*}{ Process } & \multicolumn{5}{|c|}{$g_{a A B}^{(1)}$} \\
\hline & \multirow{2}{*}{ KSVZ } & \multicolumn{4}{|c|}{ DFSZ } \\
\hline & & general & $\sin ^{2} \beta=0$ & $\sin ^{2} \beta=\frac{2}{3}$ & $\sin ^{2} \beta=1$ \\
\hline$\Sigma^{+} \rightarrow \Sigma^{+}+a$ & $-0.543(34)$ & $-0.690(36)+0.430(21) \sin ^{2} \beta$ & $-0.690(36)$ & $-0.404(36)$ & $-0.261(38)$ \\
\hline$\Sigma^{-} \rightarrow \Sigma^{-}+a$ & $-0.242(21)$ & $-0.095(29)-0.158(21) \sin ^{2} \beta$ & $-0.095(29)$ & $-0.201(23)$ & $-0.254(22)$ \\
\hline$\Sigma^{0} \rightarrow \Sigma^{0}+a$ & $-0.396(25)$ & $-0.400(29)+0.143(12) \sin ^{2} \beta$ & $-0.400(29)$ & $-0.305(27)$ & $-0.257(27)$ \\
\hline$p \rightarrow p+a$ & $-0.430(36)$ & $-0.577(38)+0.430(21) \sin ^{2} \beta$ & $-0.577(38)$ & $-0.291(38)$ & $-0.147(39)$ \\
\hline$\Xi^{-} \rightarrow \Xi^{-}+a$ & $0.140(15)$ & $0.287(25)-0.158(21) \sin ^{2} \beta$ & $0.287(25)$ & $0.181(17)$ & $0.128(16)$ \\
\hline$n \rightarrow n+a$ & $-0.002(30)$ & $0.269(34)-0.406(21) \sin ^{2} \beta$ & $0.269(34)$ & $-0.002(31)$ & $-0.138(22)$ \\
\hline$\Xi^{0} \rightarrow \Xi^{0}+a$ & $0.267(23)$ & $0.531(29)-0.406(21) \sin ^{2} \beta$ & $0.531(29)$ & $0.267(25)$ & $0.131(26)$ \\
\hline$\Lambda \rightarrow \Lambda+a$ & $0.126(25)$ & $0.310(29)-0.233(12) \sin ^{2} \beta$ & $0.310(29)$ & $0.155(27)$ & $0.077(26)$ \\
\hline $\begin{array}{l}\Sigma^{0} \rightarrow \Lambda+a \\
\Lambda \rightarrow \Sigma^{0}+a\end{array}$ & $-0.153(10)$ & $-0.308(13)+0.309(16) \sin ^{2} \beta$ & $-0.308(13)$ & $-0.102(11)$ & $0.000(13)$ \\
\hline
\end{tabular}

Table 1. Leading order axion-baryon couplings $g_{a A B}^{(1)}$ for the KSVZ axion and the DFSZ axion.

as being always

$$
\Delta g_{a A B}^{(1), \sin ^{2} \beta=1}=\frac{2-4 z-w}{3(1+z+w)}\left(\Delta q_{1}-\Delta q_{2}\right) \approx 0.008\left(\Delta q_{1}-\Delta q_{2}\right),
$$

where $A B$ here denotes particles of the same strangeness, and $\Delta q_{1}$ and $\Delta q_{2}$ depends on the quark content of these particles, i.e. $\left(\Delta q_{1}-\Delta q_{2}\right)=(\Delta s-\Delta u)$ in the case of the $\Sigma$ baryons, $\left(\Delta q_{1}-\Delta q_{2}\right)=(\Delta d-\Delta u)$ in the case of the nucleons, and $\left(\Delta q_{1}-\Delta q_{2}\right)=(\Delta d-\Delta s)$ in the case of the $\Xi$ particles (note that in cases with $\Sigma^{0}$ an additional factor $1 / 2$ and corrections from $\epsilon \neq 0$ appear in eq. (4.7)).

\subsection{Loop corrections and estimation of the NNLO LECs}

As stated already, the results for the leading order axion-nucleon coupling in the $N_{f}=3$ case are entirely in line with the results of the $N_{f}=2$ case, which is also true for the $\mathcal{O}\left(p^{2}\right)$ and $\mathcal{O}\left(p^{3}\right)$ corrections stemming from the expansion in $1 / m_{B}$ that appear in the non-relativistic heavy baryon limit, with the only exception that the nucleon mass in the chiral limit $m_{0}$ appearing in the $\mathrm{SU}(2)$ case is substituted by the average baryon mass in the chiral limit $m_{B}$. In the limit of soft axions, i.e. $q_{0} \rightarrow 0$, these terms, see eq. (3.14), rapidly vanish.

The more significant corrections stem from the one-meson loop contributions, eq. (3.38). For the calculation of the corresponding matrix elements, we use the physical meson masses and decay constants $[98,99], M_{\pi^{ \pm}}=139.57 \mathrm{MeV}, M_{\pi^{0}}=134.98 \mathrm{MeV}, M_{K^{ \pm}}=493.68 \mathrm{MeV}$, $M_{K^{0}}=497.61 \mathrm{MeV}, M_{\eta}=547.86 \mathrm{MeV}, F_{\pi}=92.1(6) \mathrm{MeV}, F_{K}=110.3(5) \mathrm{MeV}$, and $F_{\eta}=$ $118(9) \mathrm{MeV}$. Inserting this numerical input, yields

$$
\begin{gathered}
g_{a \Sigma^{+} \Sigma^{+}}^{\text {loop }}=0.096(7)-0.148(10) X_{u}-0.003(5) X_{d}+0.202(16) X_{s} \\
g_{a \Sigma^{-} \Sigma^{-}}^{\text {loop }}=0.046(6)-0.003(5) X_{u}-0.148(10) X_{d}+0.202(16) X_{s}, \\
g_{a \Sigma^{0} \Sigma^{0}}^{\text {lop }}=0.072(7)-0.076(8) X_{u}-0.076(8) X_{d}+0.202(16) X_{s},
\end{gathered}
$$




\begin{tabular}{|c|c|c|r|r|r|}
\hline \multirow{2}{*}{ Process } & \multicolumn{5}{|c|}{$g_{A B}^{\text {loop }}$} \\
\cline { 2 - 6 } & \multirow{2}{*}{ KSVZ } & \multicolumn{4}{|c|}{ DFSZ } \\
\cline { 3 - 6 } & & general & $\sin ^{2} \beta=0$ & $\sin ^{2} \beta=\frac{2}{3}$ & $\sin ^{2} \beta=1$ \\
\hline$\Sigma^{+} \rightarrow \Sigma^{+}+a$ & $0.096(7)$ & $0.162(9)-0.116(7) \sin ^{2} \beta$ & $0.162(9)$ & $0.085(8)$ & $0.046(8)$ \\
$\Sigma^{-} \rightarrow \Sigma^{-}+a$ & $0.046(6)$ & $0.064(9)-0.019(7) \sin ^{2} \beta$ & $0.064(9)$ & $0.051(6)$ & $0.045(8)$ \\
$\Sigma^{0} \rightarrow \Sigma^{0}+a$ & $0.072(7)$ & $0.114(9)-0.068(7) \sin ^{2} \beta$ & $0.114(9)$ & $0.069(8)$ & $0.046(8)$ \\
$p \rightarrow p+a$ & $0.046(5)$ & $0.100(6)-0.094(5) \sin ^{2} \beta$ & $0.100(6)$ & $0.038(6)$ & $0.007(6)$ \\
$\Xi^{-} \rightarrow \Xi^{-}+a$ & $-0.112(8)$ & $-0.122(11)+0.038(8) \sin ^{2} \beta$ & $-0.122(11)$ & $-0.096(8)$ & $-0.083(8)$ \\
$n \rightarrow n+a$ & $-0.028(5)$ & $-0.046(7)+0.051(5) \sin ^{2} \beta$ & $-0.046(7)$ & $-0.012(6)$ & $0.05(6)$ \\
$\Xi^{0} \rightarrow \Xi^{0}+a$ & $-0.145(11)$ & $-0.186(13)+0.102(8) \sin ^{2} \beta$ & $-0.186(13)$ & $-0.118(12)$ & $-0.084(12)$ \\
$\Lambda \rightarrow \Lambda+a$ & $-0.095(7)$ & $-0.112(8)+0.050(5) \sin ^{2} \beta$ & $-0.112(8)$ & $-0.079(7)$ & $-0.062(8)$ \\
\hline$\Sigma^{0} \rightarrow \Lambda+a$ & $0.030(3)$ & $0.060(4)-0.059(3) \sin ^{2} \beta$ & $0.060(4)$ & $0.020(3)$ & $0.001(4)$ \\
$\Lambda \rightarrow \Sigma^{0}+a$ & & & & & \\
\hline
\end{tabular}

Table 2. One-meson loop contributions to the axion-baryon couplings $g_{A B}^{\text {loop }}$, eq. (3.38), for the KSVZ axion and the DFSZ axion calculated at the scale $\lambda=1 \mathrm{GeV}$.

$$
\begin{aligned}
g_{a p p}^{\text {loop }} & =0.046(5)-0.119(11) X_{u}+0.098(7) X_{d}+0.064(8) X_{s}, \\
g_{a \Xi^{-} \Xi^{-}}^{\text {loop }} & =-0.112(8)+0.086(8) X_{u}+0.182(16) X_{d}-0.211(16) X_{s}, \\
g_{a n n}^{\text {loop }} & =-0.028(5)+0.098(7) X_{u}-0.119(11) X_{d}+0.064(8) X_{s}, \\
g_{a \Xi^{0} \Xi^{0}}^{\text {loop }} & =-0.145(11)+0.182(16) X_{u}+0.086(8) X_{d}-0.211(16) X_{s}, \\
g_{a \Lambda \Lambda}^{\text {loop }} & =-0.095(7)+0.099(9) X_{u}+0.099(9) X_{d}-0.149(9) X_{s}, \\
g_{a \Sigma^{0} \Lambda}^{\text {loop }} & =0.030(3)-0.089(7) X_{u}+0.089(7) X_{d}+0.000(1) X_{s},
\end{aligned}
$$

where we have set the scale at $\lambda=1 \mathrm{GeV}$. The corresponding results for the KSVZ and DFSZ models are displayed in table 2. As expected, the loop contributions are indeed subleading, where the orders of magnitude of the individual terms range between low $\mathcal{O}\left(10^{-1}\right)$ and $\mathcal{O}\left(10^{-2}\right)$. Note that just using $F_{\pi}$ or $F_{p}$ for all the decay constants does not lead to any notable change in these results since the formal difference is of higher order, $\mathcal{O}\left(p^{5}\right)$, in the chiral expansion.

It is remarkable that the loop corrections to the axion-proton vertex are about one tenth of the full $\mathrm{SU}(2)$ result [58], which shows that in this particular case the three-flavor expansion works similar to the case of the magnetic moments [100], but different to the baryon masses [101] or weak hyperon decays [102]. Consequently, the largest uncertainty in these calculations is related to the values of the LECs, as discussed next.

As for the tree-level contributions from the NNLO Lagrangian (see section 3.4), we stated already that the values of the involved LECs are undetermined hitherto. The scaledependent parts $\propto d_{k}^{r}(\lambda)$ are expected to compensate the scale-dependence of the loop contributions, such that the actual observable, $g_{a A B}$, remains scale-independent at $\mathcal{O}\left(p^{3}\right)$. For the following estimation of these hitherto unknown LECs, we therefore only consider the scale-independent part such that we can leave aside the scale-dependent loop contributions in our final estimation of the axion-baryon coupling at $\mathcal{O}\left(p^{3}\right)$. Our understanding of the problem is a Bayesian one: while formally each LEC may take on any arbitrary value, we 
nevertheless expect that with sufficient probability the LECs are restricted to values that lead to NNLO contributions to $g_{a A B}$ of roughly the same order as the loop contributions discussed above. In other words: we assume that these contributions are indeed subleading in comparison to the leading order contributions, which is basically a naturalness argument $[103,104]$. That this assumption is justified in the present case directly follows from the numerical results of the loop corrections discussed before. This argument is of course not universally valid, but it is nevertheless appropriate in a Bayesian sense, meaning that our results from this ansatz can be used as priors in future determinations of the LECs once suitable experimental or lattice QCD data is available for fitting procedures. Although it is not expected that the axion-nucleon coupling will be measured experimentally with sufficient accuracy in the near future, one may use lattice QCD to compute the relevant couplings by introducing an external isoscalar axial source into the QCD action to mimic the axion, and compute the corresponding form factors.

In practice, we performed a Monte Carlo sampling of the ten involved LECs $d_{41}^{r}, \ldots$, $d_{47}^{r}$ and $d_{43}^{i}, d_{44}^{i}$, and $d_{46}^{i}$ within a reasonable range of $\mathcal{O}\left(1 \mathrm{GeV}^{-2}\right)$ and extracted those sets of LECs that lead to NNLO corrections to $g_{a A B}$ of low $\mathcal{O}\left(10^{-1}\right)$. In particular, we set 0.15 as a numerical constraint, which is rather conservative (in view of the loop contributions given above). The allowed regions for the values of the respective LECs are then given by probability distributions of Gaußian type centered around zero (as the overall sign of the NNLO corrections is in principle undetermined from this method). With this approach, one obtains

$$
\begin{array}{ll}
d_{41}^{r}=0.00(4) \mathrm{GeV}^{-2}, & d_{42}^{r}=0.00(4) \mathrm{GeV}^{-2}, \\
d_{43}^{r}=0.00(4) \mathrm{GeV}^{-2}, & d_{43}^{i, r}=0.00(11) \mathrm{GeV}^{-2}, \\
d_{44}^{r}=0.00(6) \mathrm{GeV}^{-2}, & d_{44}^{i, r}=0.00(17) \mathrm{GeV}^{-2}, \\
d_{45}^{r}=0.00(6) \mathrm{GeV}^{-2}, & d_{46}^{r}=0.00(11) \mathrm{GeV}^{-2}, \\
d_{46}^{i, r}=0.00(14) \mathrm{GeV}^{-2}, & d_{47}^{r}=0.00(14) \mathrm{GeV}^{-2} .
\end{array}
$$

Moreover, one finds that some of the extrapolated probability distributions of the LECs are correlated. The most important correlation coefficients are given by

$$
\begin{array}{ll}
\operatorname{corr}\left(d_{41}^{r}, d_{44}^{r}\right)=0.72, & \operatorname{corr}\left(d_{41}^{r}, d_{46}^{r}\right)=-0.59, \\
\operatorname{corr}\left(d_{41}^{r}, d_{47}^{r}\right)=-0.84, & \operatorname{corr}\left(d_{44}^{r}, d_{46}^{r}\right)=-0.89, \\
\operatorname{corr}\left(d_{44}^{r}, d_{47}^{r}\right)=-0.85, & \operatorname{corr}\left(d_{46}^{r}, d_{47}^{r}\right)=0.70, \\
\operatorname{corr}\left(d_{42}^{r}, d_{45}^{r}\right)=-0.85, & \operatorname{corr}\left(d_{44}^{i, r}, d_{46}^{i, r}\right)=-0.84,
\end{array}
$$

while all other correlation coefficients are negligibly small.

If one additionally considers the large- $N_{c}$ approach, where $N_{c}$ is the number of colors, it is to be expected that the LECs for the terms with two flavor traces $\left(d_{45,46,47}^{r}\right.$ and $\left.d_{46}^{i, r}\right)$ are suppressed relative to those with only one flavor trace $\left(d_{41,42,43,44}^{r}\right.$ and $\left.d_{43,44}^{i, r}\right)$ by $\mathcal{O}\left(1 / N_{c}\right)$; see, e.g., refs. $[105,106]$. Therefore, we performed another Monte Carlo sampling for the LECs, where this expectation is taken into account, namely by assigning a larger 
probability to such sets obeying this expected rule in comparison to sets of LECs deviating from it, which are considered less probable. The result is

$$
\begin{array}{ll}
d_{41}^{r}=0.00(3) \mathrm{GeV}^{-2}, & d_{42}^{r}=0.00(2) \mathrm{GeV}^{-2}, \\
d_{43}^{r}=0.00(4) \mathrm{GeV}^{-2}, & d_{43}^{i, r}=0.00(10) \mathrm{GeV}^{-2}, \\
d_{44}^{r}=0.00(3) \mathrm{GeV}^{-2}, & d_{44}^{i, r}=0.00(13) \mathrm{GeV}^{-2}, \\
d_{45}^{r}=0.00(1) \mathrm{GeV}^{-2}, & d_{46}^{r}=0.00(1) \mathrm{GeV}^{-2}, \\
d_{46}^{i, r}=0.00(7) \mathrm{GeV}^{-2}, & d_{47}^{r}=0.00(1) \mathrm{GeV}^{-2} .
\end{array}
$$

As expected, the probability distributions of the rather suppressed LECs become considerably thinner, even though it is not excluded that they in reality may achieve higher values. For the following estimation of the axion-baryon coupling at NNLO, however, we stick to the less rigid estimation of the LECs given in eq. (4.9).

The last contribution to the $\mathcal{O}\left(q^{3}\right)$ axion-baryon coupling stem from terms $\propto \chi_{-}$ discussed in section 3.3. From the matching with $\mathrm{SU}(2)$, eq. (3.18), we deduce that

$$
\left(d_{18}+2 d_{19}\right)_{\mathrm{SU}(2)}=\left(d_{2}+3 d_{3}\right)\left(1+\frac{w}{1+z}\right)^{-1},
$$

where neither the value of the LEC $d_{19}$ from the $\mathrm{SU}(2)$ case, nor the values of $d_{2}$ and $d_{3}$ from the $\mathrm{SU}(3)$ case are known. The LEC $d_{18}$ is fixed by the Goldberger-Treiman discrepancy and given by [107]

$$
d_{18}=-0.44(24) \mathrm{GeV}^{-2} .
$$

Using this matching with $\mathrm{SU}(2)$ and applying the same Bayesian approach as described above, we extrapolate

$$
d_{2}=-0.45(24) \mathrm{GeV}^{-2}, \quad d_{3}=0.15(92) \mathrm{GeV}^{-2} .
$$

Finally, we can collect all contributions to estimate the full $\mathcal{O}\left(p^{3}\right)$ axion-baryon couplings given by

$$
g_{a A B}=g_{A B}+\frac{4 M_{\pi^{ \pm}}^{2} z}{1+z}\left(\hat{d}_{A B}^{r}-\frac{d_{2}+3 d_{3}}{1+z+w} \delta_{A B}\right)-\frac{1}{27} g_{A B}^{\text {loop }, r}
$$

which results in

$$
\begin{aligned}
& g_{a \Sigma^{+} \Sigma^{+}}=-0.547(84)+0.850(98) X_{u}-0.032(88) X_{d}-0.455(93) X_{s}, \\
& g_{a \Sigma^{-} \Sigma^{-}}=-0.245(80)-0.030(88) X_{u}+0.852(99) X_{d}-0.456(93) X_{s}, \\
& g_{a \Sigma^{0} \Sigma^{0}}=-0.399(78)+0.420(63) X_{u}+0.397(63) X_{d}-0.456(93) X_{s}, \\
& g_{a p p}=-0.432(86)+0.836(99) X_{u}-0.418(91) X_{d}-0.053(84) X_{s}, \\
& g_{a \Xi^{-} \Xi^{-}}=0.166(79)-0.083(84) X_{u}-0.455(91) X_{d}+0.854(97) X_{s}, \\
& g_{a n n}=0.003(83)-0.398(90) X_{u}+0.856(99) X_{d}-0.053(84) X_{s}, \\
& g_{a \Xi^{0} \Xi^{0}}=0.303(81)-0.424(91) X_{u}-0.052(84) X_{d}+0.854(96) X_{s}, \\
& g_{a \Lambda \Lambda}=0.138(87)-0.159(74) X_{u}-0.137(74) X_{d}+0.663(92) X_{s}, \\
& g_{a \Sigma^{0} \Lambda}=-0.161(24)+0.441(68) X_{u}-0.497(68) X_{d}+0.012(24) X_{s} .
\end{aligned}
$$

The corresponding results for the KSVZ and the DFSZ axion are collected in table 3. Note that while in the leading order case the uncertainties arise from the errors of the 


\begin{tabular}{|c|c|c|c|c|c|}
\hline \multirow{3}{*}{ Process } & \multicolumn{5}{|c|}{$g_{a A B}$} \\
\hline & \multirow{2}{*}{ KSVZ } & \multicolumn{4}{|c|}{ DFSZ } \\
\hline & & general & $\sin ^{2} \beta=0$ & $\sin ^{2} \beta=\frac{2}{3}$ & $\sin ^{2} \beta=1$ \\
\hline$\Sigma^{+} \rightarrow \Sigma^{+}+a$ & $-0.547(84)$ & $-0.709(94)+0.446(54) \sin ^{2} \beta$ & $-0.709(94)$ & $-0.412(88)$ & $-0.263(91)$ \\
\hline$\Sigma^{-} \rightarrow \Sigma^{-}+a$ & $-0.245(80)$ & $-0.113(92)-0.142(54) \sin ^{2} \beta$ & $-0.113(92)$ & $-0.208(84)$ & $-0.255(85)$ \\
\hline$\Sigma^{0} \rightarrow \Sigma^{0}+a$ & $-0.399(78)$ & $-0.417(87)+0.158(43) \sin ^{2} \beta$ & $-0.417(87)$ & $-0.311(80)$ & $-0.259(81)$ \\
\hline$p \rightarrow p+a$ & $-0.432(86)$ & $-0.589(96)+0.436(53) \sin ^{2} \beta$ & $-0.589(96)$ & $-0.298(90)$ & $-0.153(92)$ \\
\hline$\Xi^{-} \rightarrow \Xi^{-}+a$ & $0.166(79)$ & $0.299(91)-0.161(52) \sin ^{2} \beta$ & $0.299(91)$ & $0.192(83)$ & $0.138(84)$ \\
\hline$n \rightarrow n+a$ & $0.003(83)$ & $0.271(94)-0.400(53) \sin ^{2} \beta$ & $0.271(94)$ & $0.004(87)$ & $-0.130(88)$ \\
\hline$\Xi^{0} \rightarrow \Xi^{0}+a$ & $0.303(81)$ & $0.570(92)-0.409(52) \sin ^{2} \beta$ & $0.570(92)$ & $0.298(85)$ & $0.162(87)$ \\
\hline$\Lambda \rightarrow \Lambda+a$ & $0.138(87)$ & $0.314(96)-0.228(47) \sin ^{2} \beta$ & $0.314(96)$ & $0.161(90)$ & $0.085(90)$ \\
\hline $\begin{array}{l}\Sigma^{0} \rightarrow \Lambda+a \\
\Lambda \rightarrow \Sigma^{0}+a\end{array}$ & $-0.161(24)$ & $-0.323(33)+0.309(32) \sin ^{2} \beta$ & $-0.323(33)$ & $-0.117(30)$ & $-0.014(33)$ \\
\hline
\end{tabular}

Table 3. Axion-baryon couplings $g_{a A B}$, eq. (4.13), for the KSVZ axion and the DFSZ axion at $\mathcal{O}\left(p^{3}\right)$.

quark ratios $z$ and $w$, and the nucleon matrix elements $\Delta u, \Delta d$, and $\Delta s$, the uncertainties in the next-to-next-to-leading order case are dominated by the lack of knowledge of the involved LECs.

\section{Summary}

In this paper, we have worked out the axion-baryon coupling in SU(3) HBCHPT up to $\mathcal{O}\left(q^{3}\right)$ in the chiral power counting and found - in the case of the axion-nucleon coupling constants - good agreement with the already known results obtained in the $\mathrm{SU}(2)$ chiral approach. One of the most important outcomes of this study is that the axion-baryon coupling strengths are all of roughly the same order for all members of the baryon octet with only a few exceptions. The most prominent and well-known example is the axion-neutron coupling that might vanish in the KSVZ and the DSFZ model for particular values of $\sin ^{2} \beta$. Given the fact that the axion couples to hyperons with similar strength as it couples to nucleons (or even stronger, especially if the coupling to neutrons is suppressed), our results suggest a revision of axion emissivity of dense stellar objects such as neutron stars, where the cores might contain strange matter in large amounts, as have been proposed in the literature (see the References given in the introduction).

In this study, we considered rather "traditional" models. Tree-level axion-quark interactions, if any, are of the same order for all flavors, and there are no flavor-changing processes. Our calculations, however, can in principle be extended to flavor non-conserving processes by adjusting the matrix $\mathcal{X}_{q}$ accordingly and follow the strategy described in eq. (2.11). This then would lead to new terms in the axion-baryon interaction Lagrangian including baryon-changing processes.

The other modification of our calculations would be to consider models in which the couplings of the axion to the charm, bottom, and top quark are much stronger than the couplings to the up, down, and strange quarks. Such a model can easily lead to very strong 
axion-nucleon couplings driven by sea-quark effects (thus avoiding the problem of vanishing axion-neutron coupling), which in the end are balanced by correspondingly larger values of $f_{a}$, as has bee shown recently in ref. [108]. Such ideas are entirely compatible with our calculations: while the formulae derived in section 3 would be unaffected, the only difference would be that the numerical calculations of section 4 have to be adjusted as the terms $\propto \Delta q_{i}$ of (4.1) can not be neglected any more.

Our studies using both $\mathrm{SU}(2)$ and $\mathrm{SU}(3)$ symmetry have shown that the axion-baryon couplings for rather standard axion models are now known to good precision, but also that in the next-to-next-to-leading order case a higher precision is currently unattainable due to the lack of knowledge of some LECs. In this study, we used a Monte Carlo sampling procedure to extrapolate the most probable values of these unknown LECs, where "probable" here has to be understood in a Bayesian sense. It should thus be clear that our numerical leading order results, eq. (4.6), are more solid than the numerical estimations of the NNLO results.

Once the knowledge of the parameters in questions is enhanced, the numerical results of this work can easily be updated. However, the current uncertainties of $g_{a A B}$ are not the major concern considering the fact that the axion window in terms of $f_{a}$ is still very large. The largest uncertainties regarding the existence of axions is hence strongly linked to the uncertainty of $f_{a}$ and in the end it is not $g_{a A B}$ that counts, but $G_{a A B}=-g_{a A B} / f_{a}$, see eqs. (2.28) and (2.29). At last, we suggest that $G_{a A B}$ may be computed using lattice QCD by introducing an external isoscalar axial source to mimic the axion.

\section{Acknowledgments}

This work is supported in part by the Deutsche Forschungsgemeinschaft (DFG) and the National Natural Science Foundation of China (NSFC) through the funds provided to the Sino-German Collaborative Research Center "Symmetries and the Emergence of Structure in QCD" (NSFC Grant No. 12070131001, DFG Project-ID 196253076 — TRR 110), by the NSFC under Grants No. 11835015 and No. 12047503, by the Chinese Academy of Sciences (CAS) under Grant No. QYZDB-SSW-SYS013 and No. XDB34030000, by the CAS President's International Fellowship Initiative (PIFI) (Grant No. 2018DM0034), by the VolkswagenStiftung (Grant No. 93562), and by the EU (STRONG2020).

\section{A SU(3) generators in the physical basis and structure constants}

In the present work, we mainly make use of the physical basis [89], based on a set of traceless, non-Hermitian matrices $\tilde{\lambda}_{A}, A=\{1, \ldots, 8\}$, such that the baryon octet matrix $B$, eq. (2.15), and the pseudoscalar meson octet matrix $\Phi$, eq. (2.19), can be decomposed as

$$
\begin{aligned}
B & =\frac{1}{\sqrt{2}} \sum_{A} \tilde{\lambda}_{A} B_{A}, \\
\bar{B} & =\frac{1}{\sqrt{2}} \sum_{A} \bar{B}_{A} \tilde{\lambda}_{A}^{\dagger}, \\
\Phi & =\sum_{A} \tilde{\lambda}_{A} \Phi_{A},
\end{aligned}
$$


where the baryon fields $B_{A}$ and the meson fields $\Phi_{A}$ can directly be equated with the physical particles, i.e.

$$
\begin{array}{llll}
B_{1}=\Sigma^{+}, & B_{2}=\Sigma^{-}, & B_{3}=\Sigma^{0}, & B_{4}=p, \\
B_{5}=\Xi^{-}, & B_{6}=n, & B_{7}=\Xi^{0}, & B_{8}=\Lambda,
\end{array}
$$

and

$$
\begin{array}{llll}
\Phi_{1}=\pi^{+}, & \Phi_{2}=\pi^{-}, & \Phi_{3}=\pi^{0}, & \Phi_{4}=K^{+}, \\
\Phi_{5}=K^{-}, & \Phi_{6}=K^{0}, & \Phi_{7}=\bar{K}^{0}, & \Phi_{8}=\eta .
\end{array}
$$

The explicit form of the generators $\tilde{\lambda}_{A}$ is

$$
\begin{aligned}
& \tilde{\lambda}_{1}=\tilde{\lambda}_{2}^{\dagger}=\left(\begin{array}{ccc}
0 & \sqrt{2} & 0 \\
0 & 0 & 0 \\
0 & 0 & 0
\end{array}\right), \quad \tilde{\lambda}_{4}=\tilde{\lambda}_{5}^{\dagger}=\left(\begin{array}{ccc}
0 & 0 & \sqrt{2} \\
0 & 0 & 0 \\
0 & 0 & 0
\end{array}\right), \quad \tilde{\lambda}_{6}=\tilde{\lambda}_{7}^{\dagger}=\left(\begin{array}{ccc}
0 & 0 & 0 \\
0 & 0 & \sqrt{2} \\
0 & 0 & 0
\end{array}\right), \\
& \tilde{\lambda}_{3}=\left(\begin{array}{ccc}
\cos \epsilon+\frac{1}{\sqrt{3}} \sin \epsilon & 0 & 0 \\
0 & -\cos \epsilon+\frac{1}{\sqrt{3}} \sin \epsilon & 0 \\
0 & 0 & -\frac{2}{\sqrt{3}} \sin \epsilon
\end{array}\right), \\
& \tilde{\lambda}_{8}=\left(\begin{array}{ccc}
-\sin \epsilon+\frac{1}{\sqrt{3}} \cos \epsilon & 0 & 0 \\
0 & \sin \epsilon+\frac{1}{\sqrt{3}} \cos \epsilon & 0 \\
0 & 0 & -\frac{2}{\sqrt{3}} \cos \epsilon
\end{array}\right),
\end{aligned}
$$

where $\epsilon$ is the mixing angle given in eq. (2.17). These matrices are related to the common Gell-Mann matrices $\lambda_{a}$ by a unitary $8 \times 8$ transformation matrix $N$ via

$$
\tilde{\lambda}_{a}=\sum_{a} N_{A a} \lambda_{a}, \quad \lambda_{a}=\sum_{A} N_{A a}^{*} \tilde{\lambda}_{A}=\sum_{A} N_{A a} \tilde{\lambda}_{A}^{\dagger} .
$$

The non-zero matrix elements of $N$ are (note the sign convention that deviates from the one in [89])

$$
\begin{array}{ll}
N_{11}=N_{44}=N_{66}=\frac{1}{\sqrt{2}}, & N_{21}=N_{54}=N_{76}=\frac{1}{\sqrt{2}}, \\
N_{22}=N_{55}=N_{77}=-\frac{i}{\sqrt{2}}, & N_{12}=N_{45}=N_{67}=\frac{i}{\sqrt{2}}, \\
N_{33}=N_{88}=\cos \epsilon, & N_{38}=-N_{83}=\sin \epsilon
\end{array}
$$

and obey

$$
\sum_{a} N_{A a}^{*} N_{B a}=\delta_{A B}, \quad \sum_{A} N_{A a}^{*} N_{A b}=\delta_{a b}
$$

As the Gell-Mann matrices, the $\tilde{\lambda}_{A}$ 's are ortho-normalized to a value of 2 ,

$$
\left\langle\tilde{\lambda}_{A}^{\dagger} \tilde{\lambda}_{B}\right\rangle=2 \delta_{A B}
$$


Moreover, they satisfy the commutator and anticommutator relations

$$
\begin{aligned}
{\left[\tilde{\lambda}_{A}^{\dagger}, \tilde{\lambda}_{B}\right] } & =\sum_{C} \tilde{f}_{A B C} \tilde{\lambda}_{C}^{\dagger}=\sum_{C} \tilde{f}_{B A C} \tilde{\lambda}_{C}, \\
{\left[\tilde{\lambda}_{A}, \tilde{\lambda}_{B}\right] } & =\sum_{C} \tilde{f}_{C A B} \tilde{\lambda}_{C} \\
{\left[\tilde{\lambda}_{A}^{\dagger}, \tilde{\lambda}_{B}^{\dagger}\right] } & =\sum_{C} \tilde{f}_{C B A} \tilde{\lambda}_{C}^{\dagger}, \\
\left\{\tilde{\lambda}_{A}^{\dagger}, \tilde{\lambda}_{B}\right\} & =\frac{4}{3} \delta_{A B} \mathbb{1}+\sum_{C} \tilde{d}_{A B C} \tilde{\lambda}_{C}^{\dagger}=\frac{4}{3} \delta_{A B} \mathbb{1}+\sum_{C} \tilde{d}_{B A C} \tilde{\lambda}_{C},
\end{aligned}
$$

which gives the product rule

$$
\begin{aligned}
\tilde{\lambda}_{A}^{\dagger} \tilde{\lambda}_{B} & =\frac{2}{3} \delta_{A B} \mathbb{1}+\frac{1}{2} \sum_{C}\left(\tilde{d}_{A B C}+\tilde{f}_{A B C}\right) \tilde{\lambda}_{C}^{\dagger} \\
& =\frac{2}{3} \delta_{A B} \mathbb{1}+\frac{1}{2} \sum_{C}\left(\tilde{d}_{B A C}+\tilde{f}_{B A C}\right) \tilde{\lambda}_{C} .
\end{aligned}
$$

The structure constants defined in eq. (A.9) can be evaluated using

$$
\begin{aligned}
\tilde{f}_{A B C} & =\frac{1}{2}\left\langle\tilde{\lambda}_{A}^{\dagger}\left[\tilde{\lambda}_{B}, \tilde{\lambda}_{C}\right]\right\rangle, \\
\tilde{d}_{A B C} & =\frac{1}{2}\left\langle\tilde{\lambda}_{A}^{\dagger}\left\{\tilde{\lambda}_{B}, \tilde{\lambda}_{C}\right\}\right\rangle,
\end{aligned}
$$

and are related to the corresponding structure constants of the Gell-Mann representation via

$$
\begin{aligned}
& \tilde{f}_{A B C}=2 i \sum_{a, b, c} N_{A a}^{*} N_{B b} N_{C c} f^{a b c}, \\
& \tilde{d}_{A B C}=2 \sum_{a, b, c} N_{A a}^{*} N_{B b} N_{C c} d^{a b c} .
\end{aligned}
$$

In contrast to the structure constants $f^{a b c}\left(d^{a b c}\right)$ of the Gell-Mann representation, which are totally anti-symmetric (symmetric), the structure constants $\tilde{f}_{A B C}\left(\tilde{d}_{A B C}\right)$ of the physical basis are anti-symmetric (symmetric) in the last two indices only. Finally, there are sum rules for the structure constants:

$$
\begin{aligned}
& \sum_{C, D} \tilde{f}_{A C D} \tilde{f}_{B C D}=\sum_{C, D} \tilde{f}_{C A D} \tilde{f}_{C B D}=12 \delta_{A B}, \\
& \sum_{C, D} \tilde{d}_{A C D} \tilde{d}_{B C D}=\sum_{C, D} \tilde{d}_{C A D} \tilde{d}_{C B D}=\frac{20}{3} \delta_{A B} .
\end{aligned}
$$

Often, we also need the generators at $\epsilon=0$, and therefore it is convenient to set

$$
\begin{gathered}
\hat{\lambda}_{A}=\left.\tilde{\lambda}_{A}\right|_{\epsilon=0}, \\
\hat{f}_{A B C}=\left.\tilde{f}_{A B C}\right|_{\epsilon=0}, \\
\hat{d}_{A B C}=\left.\tilde{d}_{A B C}\right|_{\epsilon=0} .
\end{gathered}
$$


The properties eqs. (A.8)-(A.13) are then of course also valid for the $\hat{\lambda}_{A}$ 's with $\tilde{f}_{A B C}$ and $\tilde{d}_{A B C}$ replaced by $\hat{f}_{A B C}$ and $\hat{d}_{A B C}$, respectively. Note that now one has

$$
\hat{\lambda}_{3}=\lambda_{3}, \quad \hat{\lambda}_{8}=\lambda_{8} .
$$

The non-zero elements of the structure constants in this representation are

$$
\begin{aligned}
& \hat{f}_{147}=-\hat{f}_{256}=\hat{f}_{416}=-\hat{f}_{527}=\hat{f}_{624}=-\hat{f}_{715}=\sqrt{2}, \\
& \hat{f}_{443}=-\hat{f}_{553}=-\hat{f}_{663}=\hat{f}_{773}=-\hat{f}_{345}=\hat{f}_{367}=-1, \\
& \hat{f}_{448}=-\hat{f}_{558}=\hat{f}_{668}=-\hat{f}_{778}=-\hat{f}_{845}=-\hat{f}_{867}=-\sqrt{3}, \\
& \hat{f}_{113}=-\hat{f}_{223}=-\hat{f}_{312}=-2 .
\end{aligned}
$$

Similarly,

$$
\begin{aligned}
& \hat{d}_{147}=\hat{d}_{256}=\hat{d}_{416}=\hat{d}_{527}=\hat{d}_{624}=\hat{d}_{715}=\sqrt{2}, \\
& \hat{d}_{443}=\hat{d}_{553}=-\hat{d}_{663}=-\hat{d}_{773}=\hat{d}_{345}=-\hat{d}_{367}=1, \\
& \hat{d}_{118}=\hat{d}_{228}=\hat{d}_{338}=\hat{d}_{812}=\hat{d}_{833}=-\hat{d}_{888}=\frac{2}{\sqrt{3}}, \\
& \hat{d}_{448}=\hat{d}_{558}=\hat{d}_{668}=\hat{d}_{778}=\hat{d}_{845}=\hat{d}_{867}=-\frac{1}{\sqrt{3}} .
\end{aligned}
$$

\section{B Matrix elements of $g_{A B}, \hat{d}_{A B}$, and $g_{A B}^{\text {loop }}$}

Here we collect the matrix elements of the several contributions to the axion-baryon coupling. Table 4 shows the matrix elements of $g_{A B}$ as defined in eq. (3.3) in the physical basis at $\epsilon \neq 0$, see eq. (2.17).

In eqs. (B.1)-(B.9), we list the non-zero matrix elements of the next-to-next-to-leading order contributions to the axion-baryon coupling as defined in eqs. (3.23) and (3.24). Here we refrain from explicitly marking the scale dependence of the LECs $d_{k}^{(i)}(\lambda)$.

$$
\begin{aligned}
\hat{d}_{11}= & c^{(3)}\left\{\left(4 d_{41}+d_{47}\right)\left(1-\frac{1}{z}\right)+4 d_{42}\left(1+\frac{1}{z}\right)+2 d_{45}\left(1+\frac{1}{z}+\frac{1}{w}\right)\right\} \\
+ & \frac{c^{(8)}}{\sqrt{3}}\left\{4 d_{43}\left(1-\frac{1}{z}\right)+4 d_{44}\left(1+\frac{1}{z}\right)+2 d_{46}\left(1+\frac{1}{z}+\frac{1}{w}\right)\right. \\
& \left.\quad+d_{47}\left(1+\frac{1}{z}-\frac{2}{w}\right)\right\} \\
+ & c_{i}\left\{d_{43}^{i}\left(1-\frac{1}{z}\right)+d_{44}^{i}\left(1+\frac{1}{z}\right)+d_{46}^{i}\left(1+\frac{1}{z}+\frac{1}{w}\right)\right\},
\end{aligned}
$$




$$
\begin{aligned}
& \hat{d}_{22}=c^{(3)}\left\{\left(4 d_{41}+d_{47}\right)\left(1-\frac{1}{z}\right)-4 d_{42}\left(1+\frac{1}{z}\right)-2 d_{45}\left(1+\frac{1}{z}+\frac{1}{w}\right)\right\} \\
& +\frac{c^{(8)}}{\sqrt{3}}\left\{-4 d_{43}\left(1-\frac{1}{z}\right)+4 d_{44}\left(1+\frac{1}{z}\right)+2 d_{46}\left(1+\frac{1}{z}+\frac{1}{w}\right)\right. \\
& \left.+d_{47}\left(1+\frac{1}{z}-\frac{2}{w}\right)\right\} \\
& +c_{i}\left\{-d_{43}^{i}\left(1-\frac{1}{z}\right)+d_{44}^{i}\left(1+\frac{1}{z}\right)+d_{46}^{i}\left(1+\frac{1}{z}+\frac{1}{w}\right)\right\}, \\
& \hat{d}_{33}=c^{(3)}\left(4 d_{44}+d_{47}\right)\left(1-\frac{1}{z}\right) \\
& +\frac{c^{(8)}}{\sqrt{3}}\left\{4 d_{44}\left(1+\frac{1}{z}\right)+2 d_{46}\left(1+\frac{1}{z}+\frac{1}{w}\right)+d_{47}\left(1+\frac{1}{z}-\frac{2}{w}\right)\right\} \\
& +c_{i}\left\{d_{44}^{i}\left(1+\frac{1}{z}\right)+d_{46}^{i}\left(1+\frac{1}{z}+\frac{1}{w}\right)\right\} \\
& \hat{d}_{44}=c^{(3)}\left\{2\left(d_{41}+d_{43}\right)\left(1-\frac{1}{w}\right)+2\left(d_{42}+d_{44}\right)\left(1+\frac{1}{w}\right)\right. \\
& \left.+\left(d_{45}+d_{46}\right)\left(1+\frac{1}{z}+\frac{1}{w}\right)+d_{47}\left(1-\frac{1}{z}\right)\right\} \\
& +\frac{c^{(8)}}{\sqrt{3}}\left\{2\left(3 d_{41}-d_{43}\right)\left(1-\frac{1}{w}\right)+2\left(3 d_{42}-d_{44}\right)\left(1+\frac{1}{w}\right)\right. \\
& \left.+\left(3 d_{45}-d_{46}\right)\left(1+\frac{1}{z}+\frac{1}{w}\right)+d_{47}\left(1+\frac{1}{z}-\frac{2}{w}\right)\right\} \\
& +c_{i}\left\{d_{43}^{i}\left(1-\frac{1}{w}\right)+d_{44}^{i}\left(1+\frac{1}{w}\right)+d_{46}^{i}\left(1+\frac{1}{z}+\frac{1}{w}\right)\right\}, \\
& \hat{d}_{55}=c^{(3)}\left\{2\left(d_{41}-d_{43}\right)\left(1-\frac{1}{w}\right)-2\left(d_{42}-d_{44}\right)\left(1+\frac{1}{w}\right)\right. \\
& \left.-\left(d_{45}-d_{46}\right)\left(1+\frac{1}{z}+\frac{1}{w}\right)+d_{47}\left(1-\frac{1}{z}\right)\right\} \\
& +\frac{c^{(8)}}{\sqrt{3}}\left\{2\left(3 d_{41}+d_{43}\right)\left(1-\frac{1}{w}\right)-2\left(3 d_{42}+d_{44}\right)\left(1+\frac{1}{w}\right)\right. \\
& \left.-\left(3 d_{45}+d_{46}\right)\left(1+\frac{1}{z}+\frac{1}{w}\right)+d_{47}\left(1+\frac{1}{z}-\frac{2}{w}\right)\right\} \\
& +c_{i}\left\{-d_{43}^{i}\left(1-\frac{1}{w}\right)+d_{44}^{i}\left(1+\frac{1}{w}\right)+d_{46}^{i}\left(1+\frac{1}{z}+\frac{1}{w}\right)\right\},
\end{aligned}
$$




\begin{tabular}{|c|c|c|}
\hline Process & $A=B$ & $g_{A B}$ \\
\hline$\Sigma^{+} \rightarrow \Sigma^{+}+a$ & 1 & $2\left(\frac{c^{(8)}}{\sqrt{3}} D+c^{(3)} F\right)+c_{i} D^{i}$ \\
$\Sigma^{-} \rightarrow \Sigma^{-}+a$ & 2 & $2\left(\frac{c^{(8)}}{\sqrt{3}} D-c^{(3)} F\right)+c_{i} D^{i}$ \\
$\Sigma^{0} \rightarrow \Sigma^{0}+a$ & 3 & $\frac{2}{\sqrt{3}}\left(c^{(3)} \sin 2 \epsilon+c^{(8)} \cos 2 \epsilon\right) D+c_{i} D^{i}$ \\
$p \rightarrow p+a$ & 4 & $c^{(3)}(D+F)-\frac{c^{(8)}}{\sqrt{3}}(D-3 F)+c_{i} D^{i}$ \\
$\Xi^{-} \rightarrow \Xi^{-}+a$ & 5 & $c^{(3)}(D-F)-\frac{c^{(8)}}{\sqrt{3}}(D+3 F)+c_{i} D^{i}$ \\
$n \rightarrow n+a$ & 6 & $-c^{(3)}(D+F)-\frac{c^{(8)}}{\sqrt{3}}(D-3 F)+c_{i} D^{i}$ \\
$\Xi^{0} \rightarrow \Xi^{0}+a$ & 7 & $-c^{(3)}(D-F)-\frac{c^{(8)}}{\sqrt{3}}(D+3 F)+c_{i} D^{i}$ \\
$\Lambda \rightarrow \Lambda+a$ & 8 & $-\frac{2}{\sqrt{3}}\left(c^{(3)} \sin 2 \epsilon+c^{(8)} \cos 2 \epsilon\right) D+c_{i} D^{i}$ \\
\hline Process & $A \neq B$ & $g_{A B}$ \\
\hline$\Sigma^{0} \rightarrow \Lambda+a$ & $A=8, B=3$ & $\frac{2}{\sqrt{3}}\left(c^{(3)} \cos 2 \epsilon+c^{(8)} \sin 2 \epsilon\right) D$ \\
$\Lambda \rightarrow \Sigma^{0}+a$ & $A=3, B=8$ &
\end{tabular}

Table 4. Non-zero matrix elements of $g_{A B}$, eq. (3.3).

$$
\begin{aligned}
\hat{d}_{66}= & c^{(3)}\left\{-2\left(d_{41}+d_{43}\right)\left(\frac{1}{z}-\frac{1}{w}\right)-2\left(d_{42}+d_{44}\right)\left(\frac{1}{z}+\frac{1}{w}\right)\right. \\
& \left.-\left(d_{45}+d_{46}\right)\left(1+\frac{1}{z}+\frac{1}{w}\right)+d_{47}\left(1-\frac{1}{z}\right)\right\} \\
+ & \frac{c^{(8)}}{\sqrt{3}}\left\{2\left(3 d_{41}-d_{43}\right)\left(\frac{1}{z}-\frac{1}{w}\right)+2\left(3 d_{42}-d_{44}\right)\left(\frac{1}{z}+\frac{1}{w}\right)\right. \\
& \left.+\left(3 d_{45}-d_{46}\right)\left(1+\frac{1}{z}+\frac{1}{w}\right)+d_{47}\left(1+\frac{1}{z}-\frac{2}{w}\right)\right\} \\
+ & c_{i}\left\{d_{43}^{i}\left(\frac{1}{z}-\frac{1}{w}\right)+d_{44}^{i}\left(\frac{1}{z}+\frac{1}{w}\right)+d_{46}^{i}\left(1+\frac{1}{z}+\frac{1}{w}\right)\right\}, \\
\hat{d}_{77}= & c^{(3)}\left\{-2\left(d_{41}-d_{43}\right)\left(\frac{1}{z}-\frac{1}{w}\right)+2\left(d_{42}-d_{44}\right)\left(\frac{1}{z}+\frac{1}{w}\right)\right. \\
& \left.+\left(d_{45}-d_{46}\right)\left(1+\frac{1}{z}+\frac{1}{w}\right)+d_{47}\left(1-\frac{1}{z}\right)\right\} \\
+ & \frac{c^{(8)}}{\sqrt{3}}\left\{-2\left(3 d_{41}+d_{43}\right)\left(\frac{1}{z}-\frac{1}{w}\right)-2\left(3 d_{42}+d_{44}\right)\left(\frac{1}{z}+\frac{1}{w}\right)\right. \\
& \left.\quad-\left(3 d_{45}+d_{46}\right)\left(1+\frac{1}{z}+\frac{1}{w}\right)+d_{47}\left(1+\frac{1}{z}-\frac{2}{w}\right)\right\} \\
+ & c_{i}\left\{-d_{43}^{i}\left(\frac{1}{z}-\frac{1}{w}\right)+d_{44}^{i}\left(\frac{1}{z}+\frac{1}{w}\right)+d_{46}^{i}\left(1+\frac{1}{z}+\frac{1}{w}\right)\right\},
\end{aligned}
$$




$$
\begin{aligned}
\hat{d}_{88}= & c^{(3)}\left(\frac{4}{3} d_{44}+d_{47}\right)\left(1-\frac{1}{z}\right) \\
& +\frac{c^{(8)}}{\sqrt{3}}\left\{\frac{4}{3} d_{44}\left(1+\frac{1}{z}-\frac{8}{w}\right)-2 d_{46}\left(1+\frac{1}{z}+\frac{1}{w}\right)+d_{47}\left(1+\frac{1}{z}-\frac{2}{w}\right)\right\} \\
& +c_{i}\left\{\frac{1}{3} d_{44}^{i}\left(1+\frac{1}{z}+\frac{4}{w}\right)+d_{46}^{i}\left(1+\frac{1}{z}+\frac{1}{w}\right)\right\} .
\end{aligned}
$$

For the only non-diagonal matrix elements, one has $\hat{d}_{38}=\hat{d}_{83}$, which is given by

$$
\hat{d}_{38}=\frac{c^{(3)}}{\sqrt{3}}\left\{4 d_{44}\left(1+\frac{1}{z}\right)+d_{46}\left(1+\frac{1}{z}+\frac{1}{w}\right)\right\}+\left(\frac{4 c^{(8)}}{3} d_{44}+\frac{c_{i}}{\sqrt{3}} d_{44}^{i}\right)\left(1-\frac{1}{z}\right) .
$$

Eqs. (B.10)-(B.18) show the matrix elements of the finite one meson loop contributions $g_{A B}^{\text {loop, },}$, cf. eq. (3.39), of diagram (a) (figure 1). The expressions have been simplified using the leading order relations (2.21) and (2.22). Moreover, we have set $\mu_{\Phi_{C}}=M_{\Phi_{C}} /\left(4 \pi F_{p}\right)$ for any meson $\Phi_{C}$.

$$
\begin{aligned}
& g_{11}^{\text {loop }, r}=c^{(3)}\left\{-3 D\left(3 D^{2}-11 F^{2}\right)\left(\mu_{K^{ \pm}}^{2}-\mu_{K^{0}}^{2}\right)\right. \\
& \left.-F\left(D^{2}-9 F^{2}\right)\left(4 \mu_{\pi^{ \pm}}^{2}+\mu_{K^{ \pm}}^{2}+\mu_{K^{0}}^{2}\right)-24 D^{2} F \mu_{\pi^{ \pm}}^{2}\right\} \\
& +\frac{c^{(8)}}{\sqrt{3}}\left\{-D\left(D^{2}+9 F^{2}\right)\left(16 \mu_{\pi^{ \pm}}^{2}+\mu_{K^{ \pm}}^{2}+\mu_{K^{0}}^{2}\right)\right. \\
& \left.+54 D F^{2}\left(4 \mu_{\pi^{ \pm}}^{2}-\mu_{K^{ \pm}}^{2}-\mu_{K^{0}}^{2}\right)-3 F\left(7 D^{2}+9 F^{2}\right)\left(\mu_{K^{ \pm}}^{2}-\mu_{K^{0}}^{2}\right)\right\} \\
& +c_{i} D^{i}\left\{30 D F\left(\mu_{K^{ \pm}}^{2}-\mu_{K^{0}}^{2}\right)+\left(D^{2}+9 F^{2}\right)\left(4 \mu_{\pi^{ \pm}}^{2}+\mu_{K^{ \pm}}^{2}+\mu_{K^{0}}^{2}\right)\right. \\
& \left.+12 D^{2}\left(\mu_{K^{ \pm}}^{2}+\mu_{K^{0}}^{2}\right)\right\} \\
& g_{22}^{\text {loop }, r}=c^{(3)}\left\{-3 D\left(3 D^{2}-11 F^{2}\right)\left(\mu_{K^{ \pm}}^{2}-\mu_{K^{0}}^{2}\right)\right. \\
& \left.+F\left(D^{2}-9 F^{2}\right)\left(4 \mu_{\pi^{ \pm}}^{2}+\mu_{K^{ \pm}}^{2}+\mu_{K^{0}}^{2}\right)+24 D^{2} F \mu_{\pi^{ \pm}}^{2}\right\} \\
& +\frac{c^{(8)}}{\sqrt{3}}\left\{-D\left(D^{2}+9 F^{2}\right)\left(16 \mu_{\pi^{ \pm}}^{2}+\mu_{K^{ \pm}}^{2}+\mu_{K^{0}}^{2}\right)\right. \\
& \left.+54 D F^{2}\left(4 \mu_{\pi^{ \pm}}^{2}-\mu_{K^{ \pm}}^{2}-\mu_{K^{0}}^{2}\right)+3 F\left(7 D^{2}+9 F^{2}\right)\left(\mu_{K^{ \pm}}^{2}-\mu_{K^{0}}^{2}\right)\right\} \\
& +c_{i} D^{i}\left\{-30 D F\left(\mu_{K^{ \pm}}^{2}-\mu_{K^{0}}^{2}\right)+\left(D^{2}+9 F^{2}\right)\left(4 \mu_{\pi^{ \pm}}^{2}+\mu_{K^{ \pm}}^{2}+\mu_{K^{0}}^{2}\right)\right. \\
& \left.+12 D^{2}\left(\mu_{K^{ \pm}}^{2}+\mu_{K^{0}}^{2}\right)\right\}
\end{aligned}
$$




$$
\begin{aligned}
& g_{33}^{\text {loop }, r}=c^{(3)} D\left(17 D^{2}-9 F^{2}\right)\left(\mu_{K^{ \pm}}^{2}-\mu_{K^{0}}^{2}\right) \\
& +\frac{c^{(8)}}{\sqrt{3}}\left\{-D\left(D^{2}+9 F^{2}\right)\left(16 \mu_{\pi^{ \pm}}^{2}+\mu_{K^{ \pm}}^{2}+\mu_{K^{0}}^{2}\right)\right. \\
& \left.+54 D F^{2}\left(4 \mu_{\pi^{ \pm}}^{2}-\mu_{K^{ \pm}}^{2}-\mu_{K^{0}}^{2}\right)\right\} \\
& +c_{i} D^{i}\left\{\left(D^{2}+9 F^{2}\right)\left(4 \mu_{\pi^{ \pm}}^{2}+\mu_{K^{ \pm}}^{2}+\mu_{K^{0}}^{2}\right)+12 D^{2}\left(\mu_{K^{ \pm}}^{2}+\mu_{K^{0}}^{2}\right)\right\}, \\
& g_{44}^{\text {loop }, r}=c^{(3)}\left\{-D\left(D^{2}+3 F^{2}\right)\left(5 \mu_{\pi^{ \pm}}^{2}+8 \mu_{K^{ \pm}}^{2}-4 \mu_{K^{0}}^{2}\right)+60 D F^{2}\left(\mu_{K^{ \pm}}^{2}-\mu_{K^{0}}^{2}\right)\right. \\
& \left.-D^{2} F\left(11 \mu_{\pi^{ \pm}}^{2}+14 \mu_{K^{ \pm}}^{2}-10 \mu_{K^{0}}^{2}\right)-9 F^{3}\left(\mu_{\pi^{ \pm}}^{2}-2 \mu_{K^{ \pm}}^{2}-2 \mu_{K^{0}}^{2}\right)\right\} \\
& +\frac{c^{(8)}}{\sqrt{3}}\left\{-D^{3}\left(13 \mu_{\pi^{ \pm}}^{2}-8 \mu_{K^{ \pm}}^{2}-14 \mu_{K^{0}}^{2}\right)+9 D F^{2}\left(9 \mu_{\pi^{ \pm}}^{2}-4 \mu_{K^{ \pm}}^{2}-2 \mu_{K^{0}}^{2}\right)\right. \\
& \left.+3 D^{2} F\left(3 \mu_{\pi^{ \pm}}^{2}-14 \mu_{K^{ \pm}}^{2}-4 \mu_{K^{0}}^{2}\right)+27 F^{3}\left(\mu_{\pi^{ \pm}}^{2}+2 \mu_{K^{ \pm}}^{2}\right)\right\} \\
& +c_{i} D^{i}\left\{30 D F\left(\mu_{\pi^{ \pm}}^{2}-\mu_{K^{0}}^{2}\right)+\left(D^{2}+9 F^{2}\right)\left(\mu_{\pi^{ \pm}}^{2}+4 \mu_{K^{ \pm}}^{2}+\mu_{K^{0}}^{2}\right)\right. \\
& \left.+12 D^{2}\left(\mu_{\pi^{ \pm}}^{2}+\mu_{K^{0}}^{2}\right)\right\} \\
& g_{55}^{\text {loop }, r}=c^{(3)}\left\{-D\left(D^{2}+3 F^{2}\right)\left(5 \mu_{\pi^{ \pm}}^{2}+8 \mu_{K^{ \pm}}^{2}-4 \mu_{K^{0}}^{2}\right)+60 D F^{2}\left(\mu_{K^{ \pm}}^{2}-\mu_{K^{0}}^{2}\right)\right. \\
& \left.+D^{2} F\left(11 \mu_{\pi^{ \pm}}^{2}+14 \mu_{K^{ \pm}}^{2}-10 \mu_{K^{0}}^{2}\right)+9 F^{3}\left(\mu_{\pi^{ \pm}}^{2}-2 \mu_{K^{ \pm}}^{2}-2 \mu_{K^{0}}^{2}\right)\right\} \\
& +\frac{c^{(8)}}{\sqrt{3}}\left\{-D^{3}\left(13 \mu_{\pi^{ \pm}}^{2}-8 \mu_{K^{ \pm}}^{2}-14 \mu_{K^{0}}^{2}\right)+9 D F^{2}\left(9 \mu_{\pi^{ \pm}}^{2}-4 \mu_{K^{ \pm}}^{2}-2 \mu_{K^{0}}^{2}\right)\right. \\
& \left.-3 D^{2} F\left(3 \mu_{\pi^{ \pm}}^{2}-14 \mu_{K^{ \pm}}^{2}-4 \mu_{K^{0}}^{2}\right)-27 F^{3}\left(\mu_{\pi^{ \pm}}^{2}+2 \mu_{K^{ \pm}}^{2}\right)\right\} \\
& +c_{i} D^{i}\left\{-30 D F\left(\mu_{\pi^{ \pm}}^{2}-\mu_{K^{0}}^{2}\right)+\left(D^{2}+9 F^{2}\right)\left(\mu_{\pi^{ \pm}}^{2}+4 \mu_{K^{ \pm}}^{2}+\mu_{K^{0}}^{2}\right)\right. \\
& \left.+12 D^{2}\left(\mu_{\pi^{ \pm}}^{2}+\mu_{K^{0}}^{2}\right)\right\}
\end{aligned}
$$




$$
\begin{aligned}
& g_{66}^{\text {loop }, r}=c^{(3)}\left\{D\left(D^{2}+3 F^{2}\right)\left(5 \mu_{\pi^{ \pm}}^{2}-4 \mu_{K^{ \pm}}^{2}+8 \mu_{K^{0}}^{2}\right)+60 D F^{2}\left(\mu_{K^{ \pm}}^{2}-\mu_{K^{0}}^{2}\right)\right. \\
& \left.+D^{2} F\left(11 \mu_{\pi^{ \pm}}^{2}-10 \mu_{K^{ \pm}}^{2}+14 \mu_{K^{0}}^{2}\right)+9 F^{3}\left(\mu_{\pi^{ \pm}}^{2}-2 \mu_{K^{ \pm}}^{2}-2 \mu_{K^{0}}^{2}\right)\right\} \\
& +\frac{c^{(8)}}{\sqrt{3}}\left\{-D^{3}\left(13 \mu_{\pi^{ \pm}}^{2}-14 \mu_{K^{ \pm}}^{2}-8 \mu_{K^{0}}^{2}\right)+9 D F^{2}\left(9 \mu_{\pi^{ \pm}}^{2}-2 \mu_{K^{ \pm}}^{2}-4 \mu_{K^{0}}^{2}\right)\right. \\
& \left.+3 D^{2} F\left(3 \mu_{\pi^{ \pm}}^{2}-4 \mu_{K^{ \pm}}^{2}-14 \mu_{K^{0}}^{2}\right)+27 F^{3}\left(\mu_{\pi^{ \pm}}^{2}+2 \mu_{K^{0}}^{2}\right)\right\} \\
& +c_{i} D^{i}\left\{30 D F\left(\mu_{\pi^{ \pm}}^{2}-\mu_{K^{ \pm}}^{2}\right)+\left(D^{2}+9 F^{2}\right)\left(\mu_{\pi^{ \pm}}^{2}+\mu_{K^{ \pm}}^{2}+4 \mu_{K^{0}}^{2}\right)\right. \\
& \left.+12 D^{2}\left(\mu_{\pi^{ \pm}}^{2}+\mu_{K^{ \pm}}^{2}\right)\right\} \\
& g_{77}^{\text {loop }, r}=c^{(3)}\left\{D\left(D^{2}+3 F^{2}\right)\left(5 \mu_{\pi^{ \pm}}^{2}-4 \mu_{K^{ \pm}}^{2}+8 \mu_{K^{0}}^{2}\right)+60 D F^{2}\left(\mu_{K^{ \pm}}^{2}-\mu_{K^{0}}^{2}\right)\right. \\
& \left.-D^{2} F\left(11 \mu_{\pi^{ \pm}}^{2}-10 \mu_{K^{ \pm}}^{2}+14 \mu_{K^{0}}^{2}\right)-9 F^{3}\left(\mu_{\pi^{ \pm}}^{2}-2 \mu_{K^{ \pm}}^{2}-2 \mu_{K^{0}}^{2}\right)\right\} \\
& +\frac{c^{(8)}}{\sqrt{3}}\left\{-D^{3}\left(13 \mu_{\pi^{ \pm}}^{2}-14 \mu_{K^{ \pm}}^{2}-8 \mu_{K^{0}}^{2}\right)+9 D F^{2}\left(9 \mu_{\pi^{ \pm}}^{2}-2 \mu_{K^{ \pm}}^{2}-4 \mu_{K^{0}}^{2}\right)\right. \\
& \left.-3 D^{2} F\left(3 \mu_{\pi^{ \pm}}^{2}-4 \mu_{K^{ \pm}}^{2}-14 \mu_{K^{0}}^{2}\right)-27 F^{3}\left(\mu_{\pi^{ \pm}}^{2}+2 \mu_{K^{0}}^{2}\right)\right\} \\
& +c_{i} D^{i}\left\{-30 D F\left(\mu_{\pi^{ \pm}}^{2}-\mu_{K^{ \pm}}^{2}\right)+\left(D^{2}+9 F^{2}\right)\left(\mu_{\pi^{ \pm}}^{2}+\mu_{K^{ \pm}}^{2}+4 \mu_{K^{0}}^{2}\right)\right. \\
& \left.+12 D^{2}\left(\mu_{\pi^{ \pm}}^{2}+\mu_{K^{ \pm}}^{2}\right)\right\} \\
& g_{88}^{\text {loop }, r}=-5 c^{(3)} D\left(D^{2}-9 F^{2}\right)\left(\mu_{K^{ \pm}}^{2}-\mu_{K^{0}}^{2}\right) \\
& +\frac{c^{(8)}}{\sqrt{3}}\left\{D\left(D^{2}+27 F^{2}\right)\left(\mu_{K^{ \pm}}^{2}+\mu_{K^{0}}^{2}\right)+4 D^{3}\left(10 \mu_{\pi^{ \pm}}^{2}-3 \mu_{K^{ \pm}}^{2}-3 \mu_{K^{0}}^{2}\right)\right\} \\
& +c_{i} D^{i}\left\{3\left(D^{2}+9 F^{2}\right)\left(\mu_{K^{ \pm}}^{2}+\mu_{K^{0}}^{2}\right)-4 D^{2}\left(4 \mu_{\pi^{ \pm}}^{2}+\mu_{K^{ \pm}}^{2}+\mu_{K^{0}}^{2}\right)\right\} .
\end{aligned}
$$

The only non-diagonal matrix elements are given by

$$
\begin{aligned}
g_{38}^{\text {loop }, r}= & \frac{c^{(3)}}{\sqrt{3}} D\left\{\left(D^{2}-9 F^{2}\right)\left(8 \mu_{\pi^{ \pm}}^{2}-\mu_{K^{ \pm}}^{2}-\mu_{K^{0}}^{2}\right)+8 D^{2}\left(\mu_{\pi^{ \pm}}^{2}-2 \mu_{K^{ \pm}}^{2}-2 \mu_{K^{0}}^{2}\right)\right\} \\
& +\left\{c^{(8)} D\left(11 D^{2}-27 F^{2}\right)-3 \sqrt{3} c_{i} D^{i}\left(D^{2}-3 F^{2}\right)\right\}\left(\mu_{K^{ \pm}}^{2}-\mu_{K^{0}}^{2}\right)
\end{aligned}
$$

and $g_{83}^{\text {loop }, r}=g_{38}^{\text {loop }, r}$ 
Open Access. This article is distributed under the terms of the Creative Commons Attribution License (CC-BY 4.0), which permits any use, distribution and reproduction in any medium, provided the original author(s) and source are credited.

\section{References}

[1] A.A. Belavin, A.M. Polyakov, A.S. Schwartz and Y.S. Tyupkin, Pseudoparticle Solutions of the Yang-Mills Equations, Phys. Lett. B 59 (1975) 85 [INSPIRE].

[2] C.G. Callan Jr., R.F. Dashen and D.J. Gross, The Structure of the Gauge Theory Vacuum, Phys. Lett. B 63 (1976) 334 [InSPIRE].

[3] V. Baluni, CP Violating Effects in QCD, Phys. Rev. D 19 (1979) 2227 [InSPIRE].

[4] J.E. Kim and G. Carosi, Axions and the Strong CP Problem, Rev. Mod. Phys. 82 (2010) 557 [Erratum ibid. 91 (2019) 049902] [arXiv:0807.3125] [INSPIRE].

[5] C. Alexandrou et al., Neutron electric dipole moment using $N_{f}=2+1+1$ twisted mass fermions, Phys. Rev. D 93 (2016) 074503 [arXiv: 1510.05823] [INSPIRE].

[6] F.-K. Guo et al., The electric dipole moment of the neutron from $2+1$ flavor lattice QCD, Phys. Rev. Lett. 115 (2015) 062001 [arXiv:1502.02295] [INSPIRE].

[7] CPEDM collaboration, Storage Ring to Search for Electric Dipole Moments of Charged Particles - Feasibility Study, in CERN Yellow Reports: Monographs 3, CERN, Geneva Switzerland, arXiv:1912.07881 [INSPIRE].

[8] NEDM collaboration, Measurement of the permanent electric dipole moment of the neutron, Phys. Rev. Lett. 124 (2020) 081803 [arXiv:2001.11966] [INSPIRE].

[9] A.V. Smilga, QCD at $\theta \sim \pi$, Phys. Rev. D 59 (1999) 114021 [hep-ph/9805214] [InSPIRE].

[10] D. Lee, U.-G. Meißner, K.A. Olive, M. Shifman and T. Vonk, $\theta$-dependence of light nuclei and nucleosynthesis, Phys. Rev. Res. 2 (2020) 033392 [arXiv:2006.12321] [INSPIRE].

[11] R.D. Peccei and H.R. Quinn, CP Conservation in the Presence of Instantons, Phys. Rev. Lett. 38 (1977) 1440 [INSPIRE].

[12] R.D. Peccei and H.R. Quinn, Constraints Imposed by CP Conservation in the Presence of Instantons, Phys. Rev. D 16 (1977) 1791 [InSPIRE].

[13] S. Weinberg, A New Light Boson?, Phys. Rev. Lett. 40 (1978) 223 [InSPIRE].

[14] F. Wilczek, Problem of Strong P and T Invariance in the Presence of Instantons, Phys. Rev. Lett. 40 (1978) 279 [INSPIRE].

[15] J. Preskill, M.B. Wise and F. Wilczek, Cosmology of the Invisible Axion, Phys. Lett. B 120 (1983) 127 [INSPIRE].

[16] L.F. Abbott and P. Sikivie, A Cosmological Bound on the Invisible Axion, Phys. Lett. B 120 (1983) 133 [INSPIRE].

[17] M. Dine and W. Fischler, The Not So Harmless Axion, Phys. Lett. B 120 (1983) 137 [INSPIRE].

[18] J. Ipser and P. Sikivie, Are Galactic Halos Made of Axions?, Phys. Rev. Lett. 50 (1983) 925 [INSPIRE]. 
[19] M.S. Turner, Thermal Production of Not SO Invisible Axions in the Early Universe, Phys. Rev. Lett. 59 (1987) 2489 [Erratum ibid. 60 (1988) 1101] [INSPIRE].

[20] L.D. Duffy and K. van Bibber, Axions as Dark Matter Particles, New J. Phys. 11 (2009) 105008 [arXiv:0904.3346] [INSPIRE].

[21] D.J.E. Marsh, Axion Cosmology, Phys. Rept. 643 (2016) 1 [arXiv:1510.07633] [InSPIRE].

[22] P. Sikivie and Q. Yang, Bose-Einstein Condensation of Dark Matter Axions, Phys. Rev. Lett. 103 (2009) 111301 [arXiv:0901.1106] [INSPIRE].

[23] T.W. Donnelly, S.J. Freedman, R.S. Lytel, R.D. Peccei and M. Schwartz, Do Axions Exist?, Phys. Rev. D 18 (1978) 1607 [InSPIRE].

[24] F.P. Calaprice et al., Search for axion emission in the decay of excited states of ${ }^{12} C$, Phys. Rev. D 20 (1979) 2708 [INSPIRE].

[25] D.J. Bechis et al., Search for Axion Production in Low-energy Electron Bremsstrahlung, Phys. Rev. Lett. 42 (1979) 1511 [Erratum ibid. 43 (1979) 90] [INSPIRE].

[26] D.S.M. Alves and N. Weiner, A viable QCD axion in the MeV mass range, JHEP 07 (2018) 092 [arXiv: 1710.03764] [INSPIRE].

[27] J. Liu, N. McGinnis, C.E.M. Wagner and X.-P. Wang, Challenges for a QCD Axion at the $10 \mathrm{MeV}$ Scale, JHEP 05 (2021) 138 [arXiv:2102.10118] [INSPIRE].

[28] J.E. Kim, Weak Interaction Singlet and Strong CP Invariance, Phys. Rev. Lett. 43 (1979) 103 [INSPIRE].

[29] M.A. Shifman, A.I. Vainshtein and V.I. Zakharov, Can Confinement Ensure Natural CP Invariance of Strong Interactions?, Nucl. Phys. B 166 (1980) 493 [INSPIRE].

[30] M. Dine, W. Fischler and M. Srednicki, A Simple Solution to the Strong CP Problem with a Harmless Axion, Phys. Lett. B 104 (1981) 199 [INSPIRE].

[31] A.R. Zhitnitsky, On Possible Suppression of the Axion Hadron Interactions (in Russian), Sov. J. Nucl. Phys. 31 (1980) 260 [inSPIRE].

[32] J.E. Kim, Light Pseudoscalars, Particle Physics and Cosmology, Phys. Rept. 150 (1987) 1 [INSPIRE].

[33] L. Di Luzio, M. Giannotti, E. Nardi and L. Visinelli, The landscape of QCD axion models, Phys. Rept. 870 (2020) 1 [arXiv: 2003.01100] [INSPIRE].

[34] Z.-Y. Lu, M.-L. Du, F.-K. Guo, U.-G. Meißner and T. Vonk, QCD $\theta$-vacuum energy and axion properties, JHEP 05 (2020) 001 [arXiv: 2003.01625] [INSPIRE].

[35] S. Chang and K. Choi, Hadronic axion window and the big bang nucleosynthesis, Phys. Lett. $B 316$ (1993) 51 [hep-ph/9306216] [INSPIRE].

[36] P. Svrček and E. Witten, Axions In String Theory, JHEP 06 (2006) 051 [hep-th/0605206] [INSPIRE].

[37] N. Iwamoto, Axion Emission from Neutron Stars, Phys. Rev. Lett. 53 (1984) 1198 [INSPIRE].

[38] R. Mayle, J.R. Wilson, J.R. Ellis, K.A. Olive, D.N. Schramm and G. Steigman, Constraints on Axions from SN 1987a, Phys. Lett. B 203 (1988) 188 [inSPIRE].

[39] R.P. Brinkmann and M.S. Turner, Numerical Rates for Nucleon-Nucleon Axion Bremsstrahlung, Phys. Rev. D 38 (1988) 2338 [INSPIRE]. 
[40] G. Raffelt and D. Seckel, Bounds on Exotic Particle Interactions from SN 1987a, Phys. Rev. Lett. 60 (1988) 1793 [INSPIRE].

[41] M.S. Turner, Axions from SN 1987a, Phys. Rev. Lett. 60 (1988) 1797 [InSPIRE].

[42] A. Burrows, M.S. Turner and R.P. Brinkmann, Axions and SN 1987a, Phys. Rev. D 39 (1989) 1020 [INSPIRE].

[43] W. Keil, H.-T. Janka, D.N. Schramm, G. Sigl, M.S. Turner and J.R. Ellis, A Fresh look at axions and SN-1987A, Phys. Rev. D 56 (1997) 2419 [astro-ph/9612222] [INSPIRE].

[44] G.G. Raffelt, Astrophysical axion bounds, in Lecture Notes in Physics 741, Springer (2008), pp. 51-71 [hep-ph/0611350] [INSPIRE].

[45] J. Keller and A. Sedrakian, Axions from cooling compact stars, Nucl. Phys. A 897 (2013) 62 [arXiv: 1205.6940] [INSPIRE].

[46] A. Sedrakian, Axion cooling of neutron stars, Phys. Rev. D 93 (2016) 065044 [arXiv: 1512.07828] [INSPIRE].

[47] K. Hamaguchi, N. Nagata, K. Yanagi and J. Zheng, Limit on the Axion Decay Constant from the Cooling Neutron Star in Cassiopeia A, Phys. Rev. D 98 (2018) 103015 [arXiv: 1806.07151] [INSPIRE].

[48] M.V. Beznogov, E. Rrapaj, D. Page and S. Reddy, Constraints on Axion-like Particles and Nucleon Pairing in Dense Matter from the Hot Neutron Star in HESS J1731-347, Phys. Rev. C 98 (2018) 035802 [arXiv: 1806.07991] [INSPIRE].

[49] A. Sedrakian, Axion cooling of neutron stars. II. Beyond hadronic axions, Phys. Rev. D 99 (2019) 043011 [arXiv:1810.00190] [INSPIRE].

[50] J.H. Chang, R. Essig and S.D. McDermott, Supernova 1987A Constraints on Sub-GeV Dark Sectors, Millicharged Particles, the QCD Axion, and an Axion-like Particle, JHEP 09 (2018) 051 [arXiv: 1803.00993] [INSPIRE].

[51] P. Carenza, T. Fischer, M. Giannotti, G. Guo, G. Martínez-Pinedo and A. Mirizzi, Improved axion emissivity from a supernova via nucleon-nucleon bremsstrahlung, JCAP 10 (2019) 016 [Erratum JCAP 05 (2020) E01] [arXiv: 1906.11844] [INSPIRE].

[52] G.G. Raffelt, Astrophysical methods to constrain axions and other novel particle phenomena, Phys. Rept. 198 (1990) 1 [INSPIRE].

[53] M.S. Turner, Windows on the Axion, Phys. Rept. 197 (1990) 67 [INSPIRE].

[54] D.B. Kaplan, Opening the Axion Window, Nucl. Phys. B 260 (1985) 215 [inSPIRE].

[55] M. Srednicki, Axion Couplings to Matter. 1. CP Conserving Parts, Nucl. Phys. B 260 (1985) 689 [INSPIRE].

[56] H. Georgi, D.B. Kaplan and L. Randall, Manifesting the Invisible Axion at Low-energies, Phys. Lett. B 169 (1986) 73 [InSPIRE].

[57] G. Grilli di Cortona, E. Hardy, J. Pardo Vega and G. Villadoro, The QCD axion, precisely, JHEP 01 (2016) 034 [arXiv: 1511.02867] [INSPIRE].

[58] T. Vonk, F.-K. Guo and U.-G. Meißner, Precision calculation of the axion-nucleon coupling in chiral perturbation theory, JHEP 03 (2020) 138 [arXiv : 2001. 05327] [INSPIRE].

[59] N.K. Glendenning, The hyperon composition of neutron stars, Phys. Lett. B 114 (1982) 392 [INSPIRE]. 
[60] N.K. Glendenning, Neutron Stars Are Giant Hypernuclei?, Astrophys. J. 293 (1985) 470 [INSPIRE].

[61] O.V. Maxwell, Neutrino Emission Processes in Hyperon Populated Neutron Stars, Astrophys. J. 316 (1987) 691 [INSPIRE].

[62] F. Weber and M.K. Weigel, Neutron Star Properties and the Relativistic Nuclear Equation of State of Many Baryon Matter, Nucl. Phys. A 493 (1989) 549 [InSPIRE].

[63] J.R. Ellis, J.I. Kapusta and K.A. Olive, Strangeness, glue and quark matter content of neutron stars, Nucl. Phys. B 348 (1991) 345 [INSPIRE].

[64] N.K. Glendenning and S.A. Moszkowski, Reconciliation of neutron star masses and binding of the $\Lambda$ in hypernuclei, Phys. Rev. Lett. 67 (1991) 2414 [INSPIRE].

[65] R. Knorren, M. Prakash and P.J. Ellis, Strangeness in hadronic stellar matter, Phys. Rev. C 52 (1995) 3470 [nucl-th/9506016] [INSPIRE].

[66] J. Schaffner and I.N. Mishustin, Hyperon rich matter in neutron stars, Phys. Rev. C 53 (1996) 1416 [nucl-th/9506011] [INSPIRE].

[67] S. Balberg, I. Lichtenstadt and G.B. Cook, Roles of hyperons in neutron stars, Astrophys. J. Suppl. 121 (1999) 515 [astro-ph/9810361] [INSPIRE].

[68] M. Baldo, G.F. Burgio and H.J. Schulze, Hyperon stars in the Brueckner-Bethe-Goldstone theory, Phys. Rev. C 61 (2000) 055801 [nucl-th/9912066] [INSPIRE].

[69] H. Shen, Complete relativistic equation of state for neutron stars, Phys. Rev. C 65 (2002) 035802 [nucl-th/0202030] [inSPIRE].

[70] B.D. Lackey, M. Nayyar and B.J. Owen, Observational constraints on hyperons in neutron stars, Phys. Rev. D 73 (2006) 024021 [astro-ph/0507312] [INSPIRE].

[71] H. Djapo, B.-J. Schaefer and J. Wambach, On the appearance of hyperons in neutron stars, Phys. Rev. C 81 (2010) 035803 [arXiv:0811.2939] [InSPIRE].

[72] I. Bednarek, P. Haensel, J.L. Zdunik, M. Bejger and R. Manka, Hyperons in neutron-star cores and two-solar-mass pulsar, Astron. Astrophys. 543 (2012) A157 [arXiv:1111.6942] [INSPIRE].

[73] S. Weissenborn, D. Chatterjee and J. Schaffner-Bielich, Hyperons and massive neutron stars: vector repulsion and SU(3) symmetry, Phys. Rev. C 85 (2012) 065802 [Erratum ibid. 90 (2014) 019904] [arXiv: 1112.0234] [INSPIRE].

[74] T. Miyatsu, M.-K. Cheoun and K. Saito, Equation of state for neutron stars in SU(3) flavor symmetry, Phys. Rev. C 88 (2013) 015802 [arXiv: 1304.2121] [INSPIRE].

[75] L.L. Lopes and D.P. Menezes, Hypernuclear matter in a complete SU(3) symmetry group, Phys. Rev. C 89 (2014) 025805 [arXiv:1309.4173] [InSPIRE].

[76] M. Fortin, J.L. Zdunik, P. Haensel and M. Bejger, Neutron stars with hyperon cores: stellar radii and equation of state near nuclear density, Astron. Astrophys. 576 (2015) A68 [arXiv:1408.3052] [INSPIRE].

[77] M. Oertel, C. Providência, F. Gulminelli and A.R. Raduta, Hyperons in neutron star matter within relativistic mean-field models, J. Phys. G 42 (2015) 075202 [arXiv:1412.4545] [INSPIRE].

[78] T. Katayama and K. Saito, Hyperons in neutron stars, Phys. Lett. B 747 (2015) 43 [arXiv: 1501.05419] [INSPIRE]. 
[79] D. Chatterjee and I. Vidaña, Do hyperons exist in the interior of neutron stars?, Eur. Phys. J. A 52 (2016) 29 [arXiv:1510.06306] [inSPIRE].

[80] L. Tolos, M. Centelles and A. Ramos, Equation of State for Nucleonic and Hyperonic Neutron Stars with Mass and Radius Constraints, Astrophys. J. 834 (2017) 3 [arXiv: 1610.00919] [INSPIRE].

[81] L. Tolos, M. Centelles and A. Ramos, The Equation of State for the Nucleonic and Hyperonic Core of Neutron Stars, Publ. Astron. Soc. Austral. 34 (2017) e065 [arXiv: 1708.08681] [INSPIRE].

[82] R. Negreiros, L. Tolos, M. Centelles, A. Ramos and V. Dexheimer, Cooling of Small and Massive Hyperonic Stars, Astrophys. J. 863 (2018) 104 [arXiv : 1804. 00334] [InSPIRE].

[83] T.-T. Sun, S.-S. Zhang, Q.-L. Zhang and C.-J. Xia, Strangeness and $\Delta$ resonance in compact stars with relativistic-mean-field models, Phys. Rev. D 99 (2019) 023004 [arXiv: 1808.02207] [INSPIRE].

[84] D.D. Ofengeim, M.E. Gusakov, P. Haensel and M. Fortin, Bulk viscosity in neutron stars with hyperon cores, Phys. Rev. D 100 (2019) 103017 [arXiv:1911.08407] [INSPIRE].

[85] M. Fortin, A.R. Raduta, S. Avancini and C. Providência, Relativistic hypernuclear compact stars with calibrated equations of state, Phys. Rev. D 101 (2020) 034017 [arXiv: 2001. 08036] [INSPIRE].

[86] L. Tolos and L. Fabbietti, Strangeness in Nuclei and Neutron Stars, Prog. Part. Nucl. Phys. 112 (2020) 103770 [arXiv: 2002.09223] [INSPIRE].

[87] K. Choi, S.H. Im, H.J. Kim and H. Seong, Precision axion physics with running axion couplings, arXiv:2106.05816 [INSPIRE].

[88] J. Gasser, M.E. Sainio and A. Švarc, Nucleons with Chiral Loops, Nucl. Phys. B 307 (1988) 779 [INSPIRE].

[89] A. Krause, Baryon Matrix Elements of the Vector Current in Chiral Perturbation Theory, Helv. Phys. Acta 63 (1990) 3 [INSPIRE].

[90] E.E. Jenkins and A.V. Manohar, Baryon chiral perturbation theory using a heavy fermion Lagrangian, Phys. Lett. B 255 (1991) 558 [INSPIRE].

[91] V. Bernard, N. Kaiser, J. Kambor and U.-G. Meißner, Chiral structure of the nucleon, Nucl. Phys. B 388 (1992) 315 [inSPIRE].

[92] V. Bernard, N. Kaiser and U.-G. Meißner, Chiral dynamics in nucleons and nuclei, Int. J. Mod. Phys. E 4 (1995) 193 [hep-ph/9501384] [INSPIRE].

[93] G. Müller and U.-G. Meißner, Renormalization of the three flavor Lagrangian in heavy baryon chiral perturbation theory, Nucl. Phys. B 492 (1997) 379 [hep-ph/9610275] [INSPIRE].

[94] J.A. Oller, M. Verbeni and J. Prades, Meson-baryon effective chiral lagrangians to $\mathcal{O}\left(q^{3}\right)$, JHEP 09 (2006) 079 [hep-ph/0608204] [INSPIRE].

[95] M. Frink and U.-G. Meißner, On the chiral effective meson-baryon Lagrangian at third order, Eur. Phys. J. A 29 (2006) 255 [hep-ph/0609256] [InSPIRE].

[96] N. Fettes, U.-G. Meißner and S. Steininger, Pion-nucleon scattering in chiral perturbation theory. 1. Isospin symmetric case, Nucl. Phys. A 640 (1998) 199 [hep-ph/9803266] [INSPIRE]. 
[97] Flavour Lattice Averaging Group, FLAG Review 2019: Flavour Lattice Averaging Group (FLAG), Eur. Phys. J. C 80 (2020) 113 [arXiv: 1902.08191] [InSPIRE].

[98] Particle Data collaboration, Review of Particle Physics, Prog. Theor. Exp. Phys. 2020 (2020) 083C01 [INSPIRE].

[99] M. Kolesár and J. Říha, Application of Bayesian statistics to $\eta$-meson decay constant in

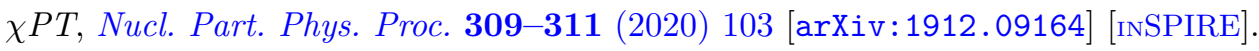

[100] U.-G. Meißner and S. Steininger, Baryon magnetic moments in chiral perturbation theory, Nucl. Phys. B 499 (1997) 349 [hep-ph/9701260] [INSPIRE].

[101] B. Borasoy and U.-G. Meißner, Chiral Expansion of Baryon Masses and $\sigma$-Terms, Annals Phys. 254 (1997) 192 [hep-ph/9607432] [InSPIRE].

[102] J. Bijnens, H. Sonoda and M.B. Wise, On the Validity of Chiral Perturbation Theory for Weak Hyperon Decays, Nucl. Phys. B 261 (1985) 185 [INSPIRE].

[103] A. Manohar and H. Georgi, Chiral Quarks and the Nonrelativistic Quark Model, Nucl. Phys. B 234 (1984) 189 [INSPIRE].

[104] M.R. Schindler and D.R. Phillips, A Bayesian approach to chiral extrapolations, PoS CD09 (2009) 019 [arXiv: 0909.3865] [INSPIRE].

[105] J. Gasser and H. Leutwyler, Chiral Perturbation Theory: Expansions in the Mass of the Strange Quark, Nucl. Phys. B 250 (1985) 465 [INSPIRE].

[106] A.V. Manohar, Large $N$ QCD, in proceedings of Les Houches Summer School in Theoretical Physics, Session 68: Probing the Standard Model of Particle Interactions, Les Houches, France, 28 July-5 September 1997, hep-ph/9802419 [INSPIRE].

[107] M. Hoferichter, J. Ruiz de Elvira, B. Kubis and U.-G. Meißner, Matching pion-nucleon Roy-Steiner equations to chiral perturbation theory, Phys. Rev. Lett. 115 (2015) 192301 [arXiv: 1507.07552] [INSPIRE].

[108] L. Darmé, L. Di Luzio, M. Giannotti and E. Nardi, Selective enhancement of the $Q C D$ axion couplings, Phys. Rev. D 103 (2021) 015034 [arXiv: 2010.15846] [InSPIRE]. 